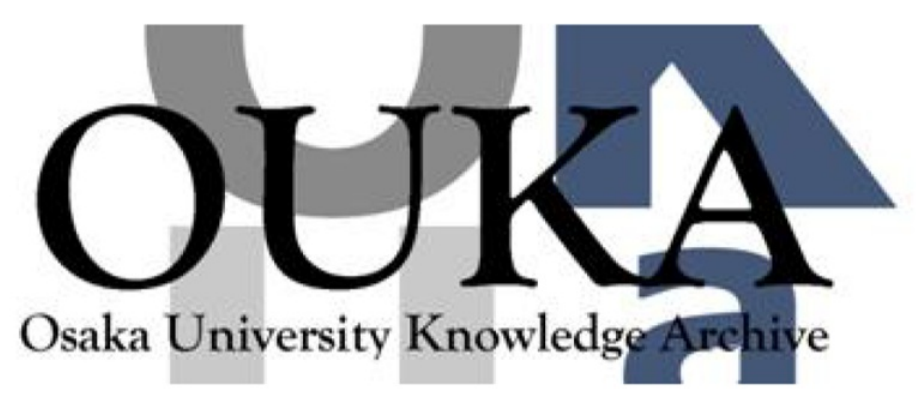

\begin{tabular}{|c|c|}
\hline Title & $\begin{array}{l}\text { Calculation of leaky Lamb waves with a semi- } \\
\text { analytical finite element method }\end{array}$ \\
\hline Author (s) & Hayashi, Takahiro; Inoue, Daisuke \\
\hline Citation & ULtrasonics. 54(6) p. 1460-p. 1469 \\
\hline Issue Date & $2014-01$ \\
\hline oaire:version & AM \\
\hline URL & https://hdl. handle. net/11094/84498 \\
\hline rights & $\begin{array}{l}\text { ( } 2014 \text { Elsevier B.V. This manuscript version is } \\
\text { made avai lable under the Creative Commons } \\
\text { Attribution-NonCommercial-NoDerivatives } 4.0 \\
\text { International License. }\end{array}$ \\
\hline Note & \\
\hline
\end{tabular}

Osaka University Knowledge Archive : OUKA

https://ir. Library. osaka-u. ac. jp/

Osaka University 


\title{
Calculation of leaky Lamb waves with a semi-analytical finite element method
}

Takahiro Hayashi and Daisuke Inoue

Graduate School of Engineering, Kyoto University, Japan

\begin{abstract}
A semi-analytical finite element method (SAFE) has been widely used for calculating dispersion curves and mode shapes of guided waves as well as transient waves in a bar like structures. Although guided wave inspection is often conducted for water-loaded plates and pipes, most of the SAFE techniques have not been extended to a plate with leaky media. This study describes leaky Lamb wave calculation with the SAFE. We formulated a new solution using a feature that a single Lamb wave mode generates a harmonic plane wave in leaky media. Dispersion curves obtained with the SAFE agreed well with the previous theoretical studies, which represents that the SAFE calculation was conducted with sufficient accuracy. Moreover, we discussed dispersion curves, attenuation curves, and displacement distributions for total transmission modes and leaky plate modes in a single side and both two side water-loaded plate.
\end{abstract}

Keywords: Leaky Lamb waves, Semi-analytical finite element method, Non-destructive evaluation

Contact author: Takahiro Hayashi: Graduate School of Engineering, Kyoto University

C3 Kyotodaigaku-katsura, Nishikyo-ku, Kyoto, Japan

+81-75-383-3797 (phone\&fax) e-mail: hayashi@kuaero.kyoto-u.ac.jp 


\section{Introduction}

When low frequency ultrasonic wave is made incident to such elongated structures as plates, pipes and rails, we can observe ultrasonic modes propagating in the longitudinal direction, called guided waves. The guided waves, which are the ultrasonic modes with resonating within the cross-section, often propagate a long distance of the order of ten or hundred meters. However, as the ultrasonic energy leaks significantly to surrounding objects such as water, anti-rust tapes, soil, and concrete, guided wave inspection is still limited in its application to existing structures [1,2].

Theoretical studies on guided waves with such energy loss have been done since long ago. In 1945, Osborne and Hart [3] derived theoretical dispersion curves in a plate with leaky fluids and presented the existence of non-damping wave appearing at a solid-fluid interface, called Scholte wave. In the late 1980's, Rokhlin et. al. [4] and Chimenti and Rokhlin [5] discussed variation of spectrum branches for various density of leaky medium. Dayal and Kinra [6] calculated and experimentally verified phase velocity dispersion curves and attenuation curves for both isotropic and anisotropic plates in water. Lowe [7] and Pavlokovic et. al. [8] showed the matrix technique can provide guided wave dispersion curves and displacement distributions even for plates and pipes with leaky media. Nowadays, the software calculating dispersion curves using the matrix technique has been widely used in the field of guided wave inspection.

A similar numerical calculation technique called a semi - analytical finite element method (SAFE) has been developed by Liu and Achenbach [9], Gavrić [10], Gry [11], Thompson and Jones [12], and authors [13-18]. Generally, the conventional calculation techniques such as finite element and finite difference methods, where whole structure is divided into sufficiently small and large number of elements, requires large amount of calculation time and memory. On the other hand, as only a cross-section 
is divided into small elements in the SAFE, calculations of guided waves propagating in a long waveguide are efficiently carried out. The SAFE also has many advantages for guided wave calculation other than the efficiency. For example, the SAFE is useful for calculating dispersion curve and analyzing wave structures for homogeneous plate like theoretical techniques as well as laminated plates and pipes, and it is feasible to calculate transient waves in such elongated structures. Therefore, many researchers have used the SAFE for guided wave calculation $[19,20]$.

Recently, the SAFE has been applied for calculating guided waves in a waveguide surrounded by leaky media. Castings and Lowe [21] and Danerjee and Kundu [22] used absorbing layers and attenuated materials for the leaky media. Mozaoti et. al. [23] modeled leaky waves radiating to infinity by using a boundary element method.

This study also deals with calculation of leaky Lamb waves using the SAFE. We formulate a new solution using a feature that a single Lamb wave mode generates a harmonic plane wave in leaky media. Moreover, we calculate dispersion curves and displacement distributions for an aluminum plate surrounded by water, and discuss some characteristic modes. 


\section{Semi-analytical finite element calculation for leaky Lamb waves}

\subsection{Governing equation in a thin plate}

Now we consider Lamb waves propagate in the $z$ direction on a cross-sectional $y-z$ plane under plane-strain conditions as shown in Fig.1, where a plate is divided into $M$ layered elements. A semi-analytical finite element method is formulated for a plate coupled with media of the densities, longitudinal and transverse velocities $\rho_{1}, c_{\mathrm{L} 1}, c_{\mathrm{T} 1}$ and $\rho_{2}, c_{\mathrm{L} 2}, c_{\mathrm{T} 2}$ on upper and lower surfaces, respectively. When the displacement, strain, stress, and external traction vectors are written as,

$$
\begin{array}{lll}
\mathbf{u}=\left(\begin{array}{ll}
u_{y} & u_{z}
\end{array}\right)^{T}, & \boldsymbol{\varepsilon}=\left(\begin{array}{lll}
\varepsilon_{y y} & \varepsilon_{z z} & \gamma_{y z}
\end{array}\right)^{T}, \\
\boldsymbol{\sigma}=\left(\begin{array}{lll}
\sigma_{y y} & \sigma_{z z} & \tau_{y z}
\end{array}\right)^{T}, & \mathbf{t}=\left(\begin{array}{ll}
t_{y} & t_{z}
\end{array}\right)^{T},
\end{array}
$$

the virtual work principle gives the following governing equations for the $j$ th elastic layered element $(j=1,2 \ldots . . M)$.

$$
\int_{C_{j}} \delta \mathbf{u}^{T} \mathbf{t} d C_{j}=\int_{A_{j}} \delta \mathbf{u}^{T}\left(-\rho_{l a y_{j}} \ddot{\mathbf{u}}\right) d A_{j}+\int_{A_{j}} \delta \boldsymbol{\varepsilon}^{T} \boldsymbol{\sigma} d A_{j}
$$

where $T$ in Eq. (1) and (2) denotes transposed matrices, $\because$ is a second differentiation with respect to time $t, \rho_{\text {lay }_{j}}$ is density in the jth layered element, $\int_{C_{j}} \bullet d C_{j}$ and $\int_{A_{j}} \bullet d A_{j}$ are the line integral on the upper and lower boundaries and the surface integral for the $j$ th layered element. The left side of the equation (2) denotes the work done by the external traction $\mathbf{t}$, and the first and second term of the right-hand side are the increment of kinetic energy and potential energy, respectively.

When considering a harmonic wave with respect to the space $z$ and time $t$, the displacement vector at an arbitrary point in the $j$ th element is expressed as,

$$
\mathbf{u}=\mathbf{N}(y) \mathbf{U}^{j} \exp \left(i \xi_{z} z-i \omega t\right)
$$

For a two-node line element, $\mathbf{N}(y)$ is the function for interpolating two values on the 
two nodes as,

$$
\mathbf{N}(y)=\left[\begin{array}{cccc}
(1-\eta) / 2 & 0 & (1+\eta) / 2 & 0 \\
0 & (1-\eta) / 2 & 0 & (1+\eta) / 2
\end{array}\right], \eta=\frac{2 y-\left(y^{j}{ }_{1}+y^{j} 2\right)}{y^{j}{ }_{2}-y^{j}{ }_{1}}
$$

where $y$ is the arbitrary position in the thickness direction within the element, $y^{j}{ }_{1}$ and $y^{j}{ }_{2}$ are the $y$ position of the lower and upper nodes of the $j$ th element, respectively. In Eq. (4), $\mathbf{U}^{j}$, the displacement vector expressing nodal displacements at $z=0$, consists of nodal displacement vectors $\mathbf{U}^{j 1}$ and $\mathbf{U}^{j 2}$ on $y^{j}{ }_{1}$ and $y^{j}{ }_{2}$ as,

$$
\mathbf{U}^{j}=\left(\begin{array}{c}
\mathbf{U}^{j 1} \\
\mathbf{U}^{j 2}
\end{array}\right), \quad \mathbf{U}^{j 1}=\left(\begin{array}{c}
U_{y}{ }^{j 1} \\
U_{z}{ }^{j 1}
\end{array}\right), \quad \mathbf{U}^{j 2}=\left(\begin{array}{c}
U_{y}{ }^{j 2} \\
U_{z}{ }^{j 2}
\end{array}\right)
$$

The strain vector is written with the strain-displacement relation as,

$$
\boldsymbol{\varepsilon}=\left[\mathbf{L}_{y} \frac{\partial}{\partial y}+\mathbf{L}_{z} \frac{\partial}{\partial z}\right] \mathbf{u}, \quad \mathbf{L}_{y}=\left[\begin{array}{ll}
1 & 0 \\
0 & 0 \\
0 & 1
\end{array}\right], \quad \mathbf{L}_{z}=\left[\begin{array}{ll}
0 & 0 \\
0 & 1 \\
1 & 0
\end{array}\right] \text {. }
$$

Substituting Eq. (3) into Eq. (6) yields

$$
\boldsymbol{\varepsilon}=\left(\mathbf{B}_{1}+i \xi_{z} \mathbf{B}_{2}\right) \mathbf{U}^{j} \exp \left(i \xi_{z} z-i \omega t\right), \quad \mathbf{B}_{1}=\mathbf{L}_{y} \mathbf{N}_{, y}, \quad \mathbf{B}_{2}=\mathbf{L}_{z} \mathbf{N}
$$

where $\mathbf{N}_{, y}$ is the differentiation of the interpolation function $\mathbf{N}_{y}$ with respect to $y$, giving,

$$
\mathbf{N}_{, y}=\frac{1}{2} \frac{\partial \eta}{\partial y}\left[\begin{array}{cccc}
-1 & 0 & 1 & 0 \\
0 & -1 & 0 & 1
\end{array}\right], \frac{\partial \eta}{\partial y}=\frac{2}{y^{j}{ }_{2}-y_{1}^{j}}
$$

The stress vector $\boldsymbol{\sigma}$ is written with the stress-strain relation as

$$
\boldsymbol{\sigma}=\mathbf{c} \boldsymbol{\varepsilon}
$$

where $\mathbf{c}$ is an elastic coefficient matrix $(3 \times 3)$. Like the displacement vector $\mathbf{u}$ in Eq. (3), the external traction vector is described using the nodal external traction vector $\mathbf{T}^{j}$ as,

$$
\mathbf{t}=\mathbf{N} \mathbf{T}^{j} \exp \left(i \xi_{z} z-i \omega t\right)
$$


Letting an imaginary displacement $\delta \mathbf{u}$ be

$$
\delta \mathbf{u}=\mathbf{N} \delta \mathbf{U}^{j} \exp \left(-i \xi_{z} z+i \omega t\right)
$$

a strain by the imaginary displacement can be written as,

$$
\delta \boldsymbol{\varepsilon}=\left(\mathbf{B}_{1}-i \xi \mathbf{B}_{2}\right) \mathbf{U}^{j} \exp \left(-i \xi_{z} z+i \omega t\right)
$$

Substituting Eq. (3) - (12) into Eq. (2) gives

$$
\begin{aligned}
\delta \mathbf{U}^{j T} \int_{C_{j}} \mathbf{N}^{T} \mathbf{N} d C_{j} \mathbf{T}^{j}=\delta \mathbf{U}^{j T} \omega^{2} \int_{A_{j}} \rho_{l a y_{j}} \mathbf{N}^{T} \mathbf{N} d A_{j} \mathbf{U}^{j} \\
+\delta \mathbf{U}^{j T} \int_{A_{j}}\left(\mathbf{B}_{1}{ }^{T}-i \xi \mathbf{B}_{2}{ }^{T}\right) \mathbf{c}\left(\mathbf{B}_{1}{ }^{T}+i \xi \mathbf{B}_{2}{ }^{T}\right) d A_{j} \mathbf{U}^{j},
\end{aligned}
$$

where the surface integral in the right-hand side and the line integral in the left-hand side can be rewritten as,

$$
\int_{A_{j}} \bullet d A_{j}=\int_{z} \int_{y_{1} j_{1}}^{y^{j_{2}}} \bullet d y d z, \int_{C_{j}} \mathbf{N}^{T} \mathbf{N} d C_{j}=\int_{z} \mathbf{I} d z, \quad \mathbf{I}=\left(\begin{array}{ll}
1 & 0 \\
0 & 1
\end{array}\right) .
$$

Then, as the both sides of Eq. (13) commonly have $\delta \mathbf{U}^{j T} \int_{z} \bullet d z$, taking the inside of the integral gives the following governing equations for the $j$ th layered element.

$$
\begin{aligned}
& \mathbf{F}^{j}=\left(\mathbf{K}_{1}^{j}+i \xi_{z} \mathbf{K}_{2}{ }^{j}+\xi_{z}{ }^{2} \mathbf{K}_{3}^{j}\right) \mathbf{U}^{j}-\omega^{2} \mathbf{M}^{j} \mathbf{U}^{j} \\
& \mathbf{F}^{j}=\mathbf{T}^{j}=\left(\begin{array}{llll}
T_{y}{ }^{j 1} & T_{z}{ }^{j 1} & T_{y}{ }^{j 2} & T_{z}{ }^{j 2}
\end{array}\right)^{T} \\
& \mathbf{K}_{1}{ }^{j}=\int_{y^{j_{1}}}^{y^{j_{2}}} \mathbf{B}_{1}{ }^{T} \mathbf{c} \mathbf{B}_{1} d y, \quad \mathbf{K}_{2}{ }^{j}=\int_{y^{j_{1}}}^{y^{j_{2}}}\left(\mathbf{B}_{1}{ }^{T} \mathbf{c B}_{2}-\mathbf{B}_{2}{ }^{T} \mathbf{c} \mathbf{B}_{1}\right) d y \\
& \mathbf{K}_{3}{ }^{j}=\int_{y^{j_{1}}}^{y^{j_{2}}} \mathbf{B}_{2}{ }^{T} \mathbf{c} \mathbf{B}_{2} d y, \quad \mathbf{M}^{j}=\int_{y^{j_{1}}}^{y^{j_{2}}} \rho_{\text {lay }} \mathbf{N}^{T} \mathbf{N} d y
\end{aligned}
$$

Calculating all values in Eq. (16) with numerical integration for all elements and overlapping the values with respect to the common nodes provide the following governing equations for the total system.

$$
\left(\mathbf{K}_{1}+i \xi_{z} \mathbf{K}_{2}+\xi_{z}^{2} \mathbf{K}_{3}-\omega^{2} \mathbf{M}\right) \mathbf{U}=\mathbf{F}
$$




$$
\mathbf{U}=\left(\begin{array}{c}
\mathbf{U}^{11} \\
\mathbf{U}^{12}\left(=\mathbf{U}^{21}\right) \\
\vdots \\
\mathbf{U}^{(M-1) 2}\left(=\mathbf{U}^{M 1}\right) \\
\mathbf{U}^{M 2}
\end{array}\right), \quad \mathbf{F}=\left(\begin{array}{c}
\mathbf{T}^{11} \\
\mathbf{T}^{12}+\mathbf{T}^{21} \\
\vdots \\
\mathbf{T}^{(M-1) 2}+\mathbf{T}^{M 1} \\
\mathbf{T}^{M 2}
\end{array}\right)
$$

where, for a two-node line element, letting the number of line nodes be $N(=M+1), \mathbf{U}$ and $\mathbf{F}$ are $2 N$ vectors and $\mathbf{K}_{1}, \mathbf{K}_{2}, \mathbf{K}_{3}$, and $\mathbf{M}$ are $2 N \times 2 N$ matrices.

When the external traction is applied only on the boundaries between the plate and leaky media at $y=y_{1}$ and $y_{2}$, the nodal traction vectors from $\mathbf{T}^{12}+\mathbf{T}^{21}$ to $\mathbf{T}^{(M-1) 2}+\mathbf{T}^{M 1}$ in the external force vector $\mathbf{F}$ become zero, and letting the nodal traction applied by the leaky media at $y=y_{1}$ and $y_{2}$ be $\mathbf{T}^{\text {leak1 }}$ and $\mathbf{T}^{\text {leak2 }}$, respectively, the following relations are satisfied,

$$
\mathbf{T}^{11}+\mathbf{T}^{\text {leak1 }}=0, \quad \mathbf{T}^{M 2}+\mathbf{T}^{\text {leak } 2}=0 .
$$

The external force vector results in

$$
\mathbf{F}=\left(\begin{array}{c}
-\mathbf{T}^{\text {leak } 1} \\
0 \\
\vdots \\
0 \\
-\mathbf{T}^{\text {leak2 }}
\end{array}\right)
$$

\subsection{Leaky waves in elastic media surrounding a thin plate}

\subsubsection{General description on leaky waves in elastic media}

A harmonic wave in a plate with the wavenumber of $\xi_{z}$ generates a harmonic plane wave with the same $z$ component of wavenumber in leaky media. From this feature, ultrasonic wave propagating in lower and upper media can be represented as following equations by omitting a common term of temporal harmonic wave $\exp (-i \omega t)$. For the lower medium of $y \leq y_{1}$, 


$$
\mathbf{u}=\mathbf{A}_{\mathrm{L} 1} \exp \left(i \xi_{z} z+i \xi_{y \mathrm{~L} 1} y\right)+\mathbf{A}_{\mathrm{T} 1} \exp \left(i \xi_{z} z+i \xi_{\mathrm{yT} 1} y\right)
$$

For the upper medium of $y \geq y_{2}$,

$$
\mathbf{u}=\mathbf{A}_{\mathrm{L} 2} \exp \left(i \xi_{z} z+i \xi_{y \mathrm{~L} 2} y\right)+\mathbf{A}_{\mathrm{T} 2} \exp \left(i \xi_{z} z+i \xi_{y \mathrm{~T} 2} y\right)
$$

In these equations, $\xi_{y \mathrm{~L} 1,2}$ and $\xi_{\mathrm{yT} 1,2}$ are a $y$ direction component of the wavenumber for longitudinal (L) and transverse (T) waves in the lower (1) and upper (2) media, respectively. Letting $X=1,2$, the following relationships are satisfied in the wavenumbers,

$$
\begin{array}{ll}
\xi_{y L X}^{2}=\xi_{\mathrm{LX}}{ }^{2}-\xi_{z}{ }^{2}, & \xi_{\mathrm{yT} X}{ }^{2}=\xi_{\mathrm{TX}}{ }^{2}-\xi_{z}{ }^{2}, \\
\xi_{\mathrm{L} X}=\frac{\omega}{c_{\mathrm{LX}}}, & \xi_{\mathrm{T} X}=\frac{\omega}{c_{\mathrm{T} X}} .
\end{array}
$$

$\mathbf{A}_{\mathrm{L} 1}, \mathbf{A}_{\mathrm{T} 1}, \mathbf{A}_{\mathrm{L} 2}$, and $\mathbf{A}_{\mathrm{T} 2}$ are vibration direction vectors for longitudinal and transverse waves in the lower and upper media, respectively. As shown in Fig. 2, the vibration directions of longitudinal and transverse waves are parallel and transverse to the propagation directions. Considering wave propagation in the upper medium $\left(y \geq y_{2}\right)$, first, the vibration direction vector for longitudinal wave $\mathbf{A}_{L 2}$ is represented using the wavenumbers $\xi_{\mathrm{yL}}$ and $\xi_{z}$ as well as an arbitrary constant $A_{\mathrm{L} 2}$ as,

$$
\mathbf{A}_{\mathrm{L} 2}=\left(\begin{array}{c}
\xi_{\mathrm{y} 2} \\
\xi_{z}
\end{array}\right) A_{\mathrm{L} 2} .
$$

Similarly, using arbitrary constant $A_{\mathrm{T} 2}$, the vibration direction vector $\mathbf{A}_{\mathrm{T} 2}$ is

$$
\mathbf{A}_{\mathrm{T} 2}=\left(\begin{array}{c}
-\xi_{z} \\
\xi_{\mathrm{yT} 2}
\end{array}\right) A_{\mathrm{T} 2} .
$$

Substituting $z=0$ and $y=y_{2}$ to Eq. (22), the amplitude vector on the upper surface $\mathbf{U}^{M 2}\left(=\left(\begin{array}{ll}U_{y}{ }^{M 2} & U_{z}{ }^{M 2}\end{array}\right)^{T}\right)$ is written as, 


$$
\mathbf{U}^{M 2}=\mathbf{A}_{\mathrm{L} 2} \exp \left(\xi_{\mathrm{yL}} y_{2}\right)+\mathbf{A}_{\mathrm{T} 2} \exp \left(i \xi_{y \mathrm{~T}} y_{2}\right)
$$

From Eqs. (24) and (25), $\mathbf{U}^{M 2}$ is rewritten as,

$$
\begin{aligned}
\mathbf{U}^{M 2} & =\left(\begin{array}{c}
\xi_{y \mathrm{~L} 2} \\
\xi_{z}
\end{array}\right) A_{\mathrm{L} 2} \exp \left(i \xi_{y \mathrm{~L} 2} y_{2}\right)+\left(\begin{array}{c}
-\xi_{z} \\
\xi_{y \mathrm{~T} 2}
\end{array}\right) A_{\mathrm{T} 2} \exp \left(i \xi_{y \mathrm{~T} 2} y_{2}\right) \\
& =\left(\begin{array}{cc}
\xi_{y \mathrm{~L} 2} & -\xi_{z} \\
\xi_{z} & \xi_{y \mathrm{~T} 2}
\end{array}\right)\left(\begin{array}{cc}
\exp \left(i \xi_{y \mathrm{~L} 2} y_{2}\right) & 0 \\
0 & \exp \left(i \xi_{y \mathrm{~T} 2} y_{2}\right)
\end{array}\right)\left(\begin{array}{c}
A_{\mathrm{L} 2} \\
A_{\mathrm{T} 2}
\end{array}\right) \\
& =\mathbf{\Xi} \mathbf{E}\left(y_{2}\right)\left(\begin{array}{l}
A_{\mathrm{L} 2} \\
A_{\mathrm{T} 2}
\end{array}\right),
\end{aligned}
$$

where

$$
\boldsymbol{\Xi}=\left(\begin{array}{cc}
\xi_{\mathrm{yL} 2} & -\xi_{z} \\
\xi_{z} & \xi_{y \mathrm{~T} 2}
\end{array}\right), \quad \mathbf{E}(y)=\left(\begin{array}{cc}
\exp \left(i \xi_{y \mathrm{~L} 2} y\right) & 0 \\
0 & \exp \left(i \xi_{y \mathrm{~T} 2} y\right)
\end{array}\right) .
$$

From Eq. (27), the arbitrary constants $A_{\mathrm{L} 2}$ and $A_{\mathrm{T} 2}$ are determined as,

$$
\left(\begin{array}{l}
A_{\mathrm{L} 2} \\
A_{\mathrm{T} 2}
\end{array}\right)=\mathbf{E}\left(-y_{2}\right) \mathbf{\Xi}^{-1} \mathbf{U}^{M 2}
$$

Now, substituting Eqs. (24), (25), and (29) to Eq. (22) provides the displacement vector at an arbitrary position in the upper leaky medium $y \geq y_{2}$ as,

$$
\begin{aligned}
\mathbf{u} & =\left(\begin{array}{c}
\xi_{y \mathrm{~L} 2} \\
\xi_{z}
\end{array}\right) A_{\mathrm{L} 2} \exp \left(i \xi_{z} z+i \xi_{y \mathrm{~L} 2} y\right)+\left(\begin{array}{c}
-\xi_{z} \\
\xi_{y \mathrm{~T} 2}
\end{array}\right) A_{\mathrm{T} 2} \exp \left(i \xi_{z} z+i \xi_{y \mathrm{~T} 2} y\right) \\
& \left.\left.=\boldsymbol{\Xi} \mathbf{E}(y)\left(\begin{array}{c}
A_{\mathrm{L} 2} \\
A_{\mathrm{T} 2}
\end{array}\right) \exp i \xi_{z} z\right)=\mathbf{\Xi} \mathbf{E}(y) \mathbf{E}\left(-y_{2}\right) \mathbf{\Xi}^{-1} \mathbf{U}^{M 2} \exp i \xi_{z} z\right) \\
& \left.=\mathbf{\Xi} \mathbf{E}\left(y-y_{2}\right) \boldsymbol{\Xi}^{-1} \mathbf{U}^{M 2} \text { expi } \xi_{z} z\right) .
\end{aligned}
$$

Then, the strain vector for leaky wave is represented using the displacement vector as

$$
\boldsymbol{\varepsilon}=\left[\mathbf{L}_{y} \frac{\partial}{\partial y}+\mathbf{L}_{z} \frac{\partial}{\partial z}\right] \mathbf{u}=\left[\mathbf{L}_{y} \frac{\partial}{\partial y}+\mathbf{L}_{z} \frac{\partial}{\partial z}\right] \boldsymbol{\Xi} \mathbf{E}\left(y-y_{2}\right) \boldsymbol{\Xi}^{-1} \mathbf{U}^{M 2} \exp \left(i \xi_{z} z\right)
$$

Since the partial differentiation of $\mathbf{E}\left(y-y_{2}\right)$ with respect to $y$ is 


$$
\frac{\partial \mathbf{E}\left(y-y_{2}\right)}{\partial y}=\mathbf{G E}\left(y-y_{2}\right), \quad \mathbf{G}=\left(\begin{array}{cc}
i \xi_{y L 2} & 0 \\
0 & i \xi_{y T 2}
\end{array}\right),
$$

the strain vector becomes

$$
\begin{aligned}
\boldsymbol{\varepsilon}=\mathbf{L}_{y} \boldsymbol{\Xi} & \mathbf{G E}\left(y-y_{2}\right) \boldsymbol{\Xi}^{-1} \mathbf{U}^{M 2} \exp \left(i \xi_{z} z\right) \\
& +i \xi_{z} \mathbf{L}_{z} \boldsymbol{\Xi} \mathbf{E}\left(y-y_{2}\right) \boldsymbol{\Xi}^{-1} \mathbf{U}^{M 2} \exp \left(i \xi_{z} z\right)
\end{aligned} .
$$

Using the relationship $\mathbf{E}(0)=\left(\begin{array}{ll}1 & 0 \\ 0 & 1\end{array}\right)$ at $y=y_{2}$, the strain vector on the upper surface can be described as,

$$
\begin{aligned}
\boldsymbol{\varepsilon}\left(y=y_{2}\right) & =\mathbf{L}_{y} \boldsymbol{\Xi} \mathbf{G} \boldsymbol{\Xi}^{-1} \mathbf{U}^{M 2} \exp \left(i \xi_{z} z\right)+i \xi_{z} \mathbf{L}_{z} \mathbf{U}^{M 2} \exp \left(i \xi_{z} z\right) \\
& =\left(\mathbf{L}_{y} \boldsymbol{\Xi} \mathbf{G} \boldsymbol{\Xi}^{-1}+i \xi_{z} \mathbf{L}_{z}\right) \mathbf{U}^{M 2} \exp \left(i \xi_{z} z\right)
\end{aligned}
$$

The stress-strain relation is described using the stiffness matrix in the upper leaky medium $\mathbf{c}^{\text {leak2 as }}$

$$
\boldsymbol{\sigma}=\mathbf{c}^{\text {leak } 2} \boldsymbol{\varepsilon}
$$

As a direction normal to the leaky medium region is $-y$ at $y=y_{2}$, the surface traction that the leaky medium applies on the upper surface of the $M$ th layered element is expressed as,

$$
\begin{aligned}
\mathbf{t} & =-\left(\begin{array}{ccc}
1 & 0 & 0 \\
0 & 0 & 1
\end{array}\right)\left(\begin{array}{c}
\sigma_{y y} \\
\sigma_{z z} \\
\tau_{y z}
\end{array}\right)_{y=y_{2}}=-\mathbf{n c}^{\text {leak2}} \boldsymbol{\varepsilon}\left(y=y_{2}\right) \\
& =-\mathbf{n} \mathbf{c}^{\text {leak } 2}\left(\mathbf{L}_{y} \boldsymbol{\Xi} \mathbf{G} \boldsymbol{\Xi}^{-1}+i \xi_{z} \mathbf{L}_{z}\right) \mathbf{U}^{M 2} \exp \left(i \xi_{z} z\right),
\end{aligned}
$$

where the normal direction matrix $\mathbf{n}$ is defined as,

$$
\mathbf{n}=\left(\begin{array}{lll}
1 & 0 & 0 \\
0 & 0 & 1
\end{array}\right)
$$

Removing $\exp \left(i \xi_{z} z\right)$ from Eq. (36) provides a nodal external force vector that the leaky medium applies to the plate as, 


$$
\mathbf{T}^{\text {leak2 }}=\mathbf{P}^{M 2} \mathbf{U}^{M 2}, \mathbf{P}^{M 2}=-\mathbf{n} \mathbf{c}^{\text {leak } 2}\left(\mathbf{L}_{y} \boldsymbol{\Xi} \mathbf{G} \boldsymbol{\Xi}^{-1}+i \xi_{z} \mathbf{L}_{z}\right)
$$

Similarly, a nodal external traction vector at $y=y_{1}$ can be obtained by replacing $\xi_{y \mathrm{~L} 2}, \xi_{y \mathrm{~T} 2}$ to $\xi_{y \mathrm{~L} 1}, \xi_{y \mathrm{~T} 1},-\mathbf{n}$ to $\mathbf{n}, \mathbf{c}^{\text {leak} 2}$ to $\mathbf{c}^{\text {leak } 1}$, and $\mathbf{U}^{M 2}$ to $\mathbf{U}^{11}$ respectively as,

$$
\mathbf{T}^{\text {leak } 1}=\mathbf{P}^{11} \mathbf{U}^{11}, \quad \mathbf{P}^{11}=\mathbf{n} \mathbf{c}^{\text {leak } 1}\left(\mathbf{L}_{y} \Xi^{\prime} \mathbf{G}^{\prime} \Xi^{\prime-1}+i \xi_{z} \mathbf{L}_{z}\right)
$$

where $\boldsymbol{\Xi}^{\prime}$ and $\mathbf{G}^{\prime}$ are obtained by replacing $\xi_{\mathrm{yL} 2}$ and $\xi_{\mathrm{yT} 2}$ in $\boldsymbol{\Xi}$ and $\mathbf{G}$ to $\xi_{y \mathrm{~L} 1}$ and $\xi_{\mathrm{yT} 1}$, respectively.

Substituting Eqs. (38) and (39) to Eq. (20) gives

$$
\mathbf{F}=-\mathbf{P U}, \quad \mathbf{P}=\left(\begin{array}{ccccc}
\mathbf{P}^{11} & 0 & \cdots & 0 & 0 \\
0 & 0 & & & 0 \\
\vdots & & \ddots & & \vdots \\
0 & & & 0 & 0 \\
0 & 0 & \cdots & 0 & \mathbf{P}^{M 2}
\end{array}\right)
$$

Since the matrix $\mathbf{P}$ is a function of $\xi_{z}$, the governing equation (15) can be represented as,

$$
\left(\mathbf{K}_{1}+i \xi_{z} \mathbf{K}_{2}+\xi_{z}^{2} \mathbf{K}_{3}-\omega^{2} \mathbf{M}+\mathbf{P}\left(\xi_{z}, \omega\right)\right) \mathbf{U}=0
$$

For a certain angular frequency $\omega$, Eq. (41) is regarded as a generalized nonlinear eigenvalue problem with respect to the wavenumber $\xi_{z}$. Although a wide variety of solutions have been developed for many kinds of nonlinear eigenvalue problems [23], the simplest kind of nonlinear problem is the polynomial eigenvalue problem. Therefore, in the section 2.3, we transform Eq. (41) into the form of 3rd order polynomial eigenvalue problem under the condition that leaky media are non-viscous fluids with the same sound velocities.

2.2.2 Total transmission and leaky plate mode in liquid media 
For non-viscous fluid leaky media, $\mathbf{P}^{M 2}$ and $\mathbf{P}^{11}$ in Eq. (38) and (39) are obtained in the following simple form by setting transverse velocity in the leaky media to zero $\left(c_{\mathrm{T} 1}, c_{\mathrm{T} 2} \rightarrow 0\right)$,

$$
\mathbf{P}^{11}=\frac{i \rho_{1} \omega^{2}}{\xi_{\mathrm{yL} 1}}\left(\begin{array}{ll}
1 & 0 \\
0 & 0
\end{array}\right), \quad \mathbf{P}^{M 2}=-\frac{i \rho_{2} \omega^{2}}{\xi_{\mathrm{yL} 2}}\left(\begin{array}{ll}
1 & 0 \\
0 & 0
\end{array}\right) .
$$

Supposing sound velocities in the lower and upper media are the same, Eq. (41) can be rewritten by using the wavenumber in the $y$ direction of the leaky media $\xi_{y}\left(=\xi_{y \mathrm{~L} 1}=\xi_{y \mathrm{~L} 2}\right) \mathrm{as}$

$$
\begin{aligned}
& \left(\mathbf{K}_{1}+i \xi_{z} \mathbf{K}_{2}+\xi_{z}{ }^{2} \mathbf{K}_{3}-\omega^{2} \mathbf{M}-\frac{\omega^{2}}{\xi_{y}} \mathbf{Q}\right) \mathbf{U}=0 \\
& \mathbf{Q}=\left(\begin{array}{ccccc}
-i \rho_{1} & 0 & \cdots & 0 & 0 \\
0 & 0 & & & 0 \\
\vdots & & \ddots & & \vdots \\
0 & & & i \rho_{2} & 0 \\
0 & 0 & \cdots & 0 & 0
\end{array}\right)
\end{aligned}
$$

For positive wavenumber in the $y$ direction $\xi_{y}$, Eq. (44) represents the total transmission case when ultrasonic wave is incident from the lower fluid and leaks and radiates to the upper fluid.

Negating the sign of the wavenumber in the lower medium $\left(\xi_{y}\left(\equiv-\xi_{\mathrm{yL} 1}=\xi_{\mathrm{yL} 2}\right)\right)$ can model leaky plate modes where ultrasonic wave leaks to the both media from the plate as,

$$
\mathbf{Q}=\left(\begin{array}{ccccc}
i \rho_{1} & 0 & \cdots & 0 & 0 \\
0 & 0 & & & 0 \\
\vdots & & \ddots & & \vdots \\
0 & & & i \rho_{2} & 0 \\
0 & 0 & \cdots & 0 & 0
\end{array}\right) .
$$

Eqs. (44) and (45) can deal with leaky media with different densities, and we can calculate leaky Lamb waves in a plate coupled with fluid medium on a single side 
by setting $\rho_{1} \rightarrow 0$ or $\rho_{2} \rightarrow 0$.

\subsection{Linearization of nonlinear eigenvalue problem using the symmetry of Lamb}

\section{wave modes}

Although Eq. (43) is still a nonlinear eigenvalue problem, the equation can be deformed in the form of 3rd order polynomial eigenvalue problem using the symmetry of possible Lamb wave modes and then be linearized in the ordinary way. Letting the $m$ th eigenvalues and right eigenvector be $\xi_{y m}, \xi_{z m}$, and $\boldsymbol{\varphi}_{m}$, the nonlinear eigenvalue problem Eq. (43) satisfies

$$
\left(\mathbf{K}_{1}+i \xi_{z m} \mathbf{K}_{2}+\xi_{z m}{ }^{2} \mathbf{K}_{3}-\omega^{2} \mathbf{M}-i \frac{\omega^{2}}{\xi_{y m}} \mathbf{Q}\right) \boldsymbol{\varphi}_{m}=0,
$$

where the eigenvalues and right eigenvector correspond to the wavenumbers in the $y$ and $z$ directions and displacement distribution of the $m$ th mode. $\xi_{y m}$ and $\xi_{z m}$ satisfies the following equation like Eq. (23) as,

$$
\xi_{y m}{ }^{2}+\xi_{z m}{ }^{2}=\xi_{f}{ }^{2}
$$

Now, we divide the displacement distribution vector $\boldsymbol{\varphi}_{m}$ into two components of displacement in the $y$ and $z$ directions.

$$
\boldsymbol{\varphi}_{m}=\left(\begin{array}{c}
\boldsymbol{\varphi}_{y m} \\
\boldsymbol{\varphi}_{z m}
\end{array}\right)
$$

Then, Eq. (46) is also satisfied by changing the sigh of the wavenumber and displacement distribution vector in the $z$ direction like $\xi_{z m} \rightarrow-\xi_{z m}$ and $\boldsymbol{\varphi}_{z m} \rightarrow-\boldsymbol{\varphi}_{z m}$ in order to retain the symmetry in the $\pm z$ direction, giving

$$
\left(\mathbf{K}_{1}-i \xi_{z m} \mathbf{K}_{2}+\xi_{z m}{ }^{2} \mathbf{K}_{3}-\omega^{2} \mathbf{M}-i \frac{\omega^{2}}{\xi_{y m}} \mathbf{Q}\right)\left(\begin{array}{c}
\boldsymbol{\varphi}_{y m} \\
-\boldsymbol{\varphi}_{z m}
\end{array}\right)=0 \text {. }
$$


When matrices $\mathbf{K}_{1}, \mathbf{K}_{2}, \mathbf{K}_{3}, \mathbf{M}$, and $\mathbf{Q}$ are also divided into two parts with respect to displacements in the $y$ and $z$ directions like $\mathbf{K}_{1}=\left(\begin{array}{ll}\mathbf{K}_{1 y} & \mathbf{K}_{1 z}\end{array}\right)$, Eqs. (46) and (49) are rewritten as,

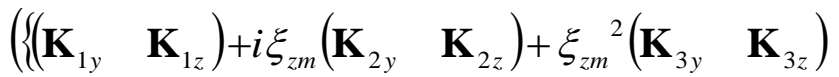

$$
\begin{aligned}
& -\omega^{2}\left(\begin{array}{ll}
\mathbf{M}_{y} & \mathbf{M}_{z}
\end{array}\right)-i \frac{\omega^{2}}{\xi_{y m}}\left(\begin{array}{ll}
\mathbf{Q}_{y} & \mathbf{Q}_{z}
\end{array}\right)\left(\begin{array}{l}
\boldsymbol{\varphi}_{y m} \\
\boldsymbol{\varphi}_{z m}
\end{array}\right)=0
\end{aligned}
$$

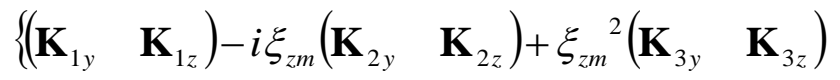

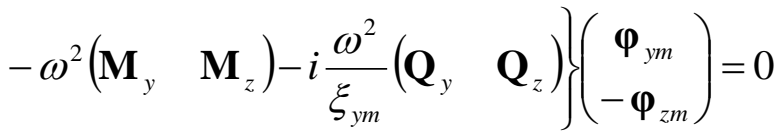

Considering $\mathbf{Q}_{z}=0$ and calculating $\{$ Eq. (50) + Eq. (51) $\} / 2+\xi_{z m}\{$ Eq. (50) - Eq. (51) $\} / 2$ yields

$$
\left(\mathbf{H}_{11}+\xi_{z m}{ }^{2} \mathbf{H}_{12}-i \frac{\omega^{2}}{\xi_{y m}} \mathbf{Q}\right)\left(\begin{array}{c}
\boldsymbol{\varphi}_{y m} \\
\xi_{z m} \boldsymbol{\varphi}_{z m}
\end{array}\right)=0,
$$

where

$$
\mathbf{H}_{11}=\left(\begin{array}{ll}
\mathbf{K}_{1 y} & \mathbf{K}_{1 z}+i \mathbf{K}_{2 z}
\end{array}\right)-\omega^{2}\left(\begin{array}{ll}
\mathbf{M}_{y} & \mathbf{M}_{z}
\end{array}\right), \quad \mathbf{H}_{12}=\left(\begin{array}{ll}
-i \mathbf{K}_{2 y}-\mathbf{K}_{3 y} & \mathbf{K}_{3 z}
\end{array}\right) .
$$

Substituting Eq. (47) to Eq. (52) and rearranging it provide the following 3rd order polynomial eigenvalue problem.

$$
\begin{aligned}
& \left(\mathbf{H}_{0}+\xi_{y m} \mathbf{H}_{1}+\xi_{y m}{ }^{3} \mathbf{H}_{3}\right) \boldsymbol{\varphi}_{m}^{\prime}=0 \\
& \mathbf{H}_{0}=-i \omega^{2} \mathbf{Q}, \quad \mathbf{H}_{1}=\mathbf{H}_{11}-\xi_{f}{ }^{2} \mathbf{H}_{12}, \quad \mathbf{H}_{3}=-\mathbf{H}_{12}, \quad \boldsymbol{\varphi}_{m}^{\prime}=\left(\begin{array}{c}
\boldsymbol{\varphi}_{y m} \\
\xi_{z m} \boldsymbol{\varphi}_{z m}
\end{array}\right)
\end{aligned}
$$

The general polynomial eigenvalue problem can be deformed to linear eigenvalue problem as, 


$$
\left[\left(\begin{array}{ccc}
\mathbf{0} & \mathbf{H}_{1} & \mathbf{H}_{0} \\
\mathbf{I} & \mathbf{0} & \mathbf{0} \\
\mathbf{0} & \mathbf{I} & \mathbf{0}
\end{array}\right)-\xi_{y m}\left(\begin{array}{ccc}
-\mathbf{H}_{3} & \mathbf{0} & \mathbf{0} \\
\mathbf{0} & \mathbf{I} & \mathbf{0} \\
\mathbf{0} & \mathbf{0} & \mathbf{I}
\end{array}\right)\right]\left(\begin{array}{c}
\xi_{y m}{ }^{2} \boldsymbol{\varphi}_{m}^{\prime} \\
\xi_{y m} \boldsymbol{\varphi}_{m}^{\prime} \\
\boldsymbol{\varphi}_{m}^{\prime}
\end{array}\right)=0
$$

$\mathbf{Q}$ and $\mathbf{H}_{0}$ have non-zero elements only in the 1st row and 1st column and in the $(2 N-1)$ th row and $(2 N-1)$ th column as shown in Eqs. (44) and (45). Removing the zero elements from Eq. (55) results in the linear eigenvalue problem consisting of $(4 N+2) \times$ $(4 N+2)$ matrices.

$$
\left[\left(\begin{array}{ccc}
\mathbf{0} & \mathbf{H}_{1} & \mathbf{H}_{0}^{\prime} \\
\mathbf{I} & \mathbf{0} & \mathbf{0} \\
\mathbf{0} & \mathbf{I}^{\prime} & \mathbf{0}
\end{array}\right)-\xi_{y m}\left(\begin{array}{ccc}
-\mathbf{H}_{3} & \mathbf{0} & \mathbf{0} \\
\mathbf{0} & \mathbf{I} & \mathbf{0} \\
\mathbf{0} & \mathbf{0} & \mathbf{I}^{\prime \prime}
\end{array}\right)\right]\left(\begin{array}{c}
\xi_{y m}{ }^{2} \boldsymbol{\varphi}_{m}^{\prime} \\
\xi_{y m} \boldsymbol{\varphi}_{m}^{\prime} \\
\boldsymbol{\varphi}_{m}^{\prime \prime}
\end{array}\right)=0
$$

where

$$
\begin{aligned}
& \mathbf{H}_{0}^{\prime}=-i \omega^{2} \mathbf{Q}^{\prime}, \quad \mathbf{Q}^{\prime}=\left(\begin{array}{cc}
\rho_{1} & 0 \\
0 & \vdots \\
\vdots & 0 \\
0 & \rho_{2} \\
0 & 0
\end{array}\right), \quad \mathbf{I}^{\prime}=\left(\begin{array}{ccccc}
1 & 0 & \cdots & 0 & 0 \\
0 & \cdots & 0 & 1 & 0
\end{array}\right), \\
& \mathbf{I}^{\prime \prime}=\left(\begin{array}{ll}
1 & 0 \\
0 & 1
\end{array}\right), \quad \boldsymbol{\varphi}_{m}^{\prime \prime}=\left(\begin{array}{c}
\phi_{m 1}^{\prime} \\
\phi_{m N-1}^{\prime}
\end{array}\right)
\end{aligned}
$$

$\phi_{m 1}^{\prime}$ and $\phi_{m N-1}^{\prime}$ in Eq. (57) are the 1 st and $(2 N-1)$ th elements of $\boldsymbol{\varphi}_{m}^{\prime}$. Solving the eigenvalue problem yields $4 N+2$ eigenvalues $\xi_{y m}$, which corresponds to the wavenumber in the $y$ direction, and right and left eigenvectors $\mathbf{v}^{R}{ }_{m}$ and $\mathbf{v}_{m}^{L}$ $(m=1,2 \ldots 4 N+2)$.

\subsection{Derivation of group velocity}

Eq. (56) can be expressed using the right and left eigenvectors $\mathbf{v}_{m}^{R}$ and $\mathbf{v}_{m}^{L}$ as,

$$
\left(\mathbf{A}-\xi_{y m} \mathbf{B}\right) \mathbf{v}^{R}{ }_{m}=0
$$




$$
\mathbf{v}_{m}^{L}\left(\mathbf{A}-\xi_{y m} \mathbf{B}\right)=0
$$

where

$$
\mathbf{A}=\left(\begin{array}{ccc}
\mathbf{0} & \mathbf{H}_{1} & \mathbf{H}_{0}^{\prime} \\
\mathbf{I} & \mathbf{0} & \mathbf{0} \\
\mathbf{0} & \mathbf{I}^{\prime} & \mathbf{0}
\end{array}\right), \quad \mathbf{B}=\left(\begin{array}{ccc}
-\mathbf{H}_{3} & \mathbf{0} & \mathbf{0} \\
\mathbf{0} & \mathbf{I} & \mathbf{0} \\
\mathbf{0} & \mathbf{0} & \mathbf{I}^{\prime \prime}
\end{array}\right)
$$

Then,

$$
\begin{aligned}
& \frac{d \mathbf{A}}{d \omega}=\left(\begin{array}{ccc}
\mathbf{0} & \frac{d \mathbf{H}_{1}}{d \omega} & \frac{d \mathbf{H}_{0}^{\prime}}{d \omega} \\
\mathbf{0} & \mathbf{0} & \mathbf{0} \\
\mathbf{0} & \mathbf{0} & \mathbf{0}
\end{array}\right)=\left(\begin{array}{ccc}
\mathbf{0} & -2 \omega \mathbf{M}-2 \frac{\xi_{f}^{2}}{\omega} \mathbf{H}_{12} & -2 i \omega \mathbf{Q} \\
\mathbf{0} & \mathbf{0} & \mathbf{0} \\
\mathbf{0} & \mathbf{0} & \mathbf{0}
\end{array}\right), \\
& \frac{d \mathbf{B}}{d \omega}=0
\end{aligned}
$$

Differentiating Eq. (58) with $\omega$ gives

$$
\left(\frac{d \mathbf{A}}{d \omega}-\frac{d \xi_{y m}}{d \omega} \mathbf{B}\right) \mathbf{v}^{R}{ }_{m}+\left(\mathbf{A}-\xi_{y m} \mathbf{B}\right) \frac{d \mathbf{v}^{R}}{d \omega}=0 .
$$

Applying $\mathbf{v}_{m}^{L}$ to this equation from the left, the second term becomes zero from Eq. (59) and the following relation can be derived.

$$
\begin{aligned}
& \mathbf{v}_{m}^{L}\left(\frac{d \mathbf{A}}{d \omega}-\frac{d \xi_{y m}}{d \omega} \mathbf{B}\right) \mathbf{v}^{R}{ }_{m}=0 \\
& \therefore \quad \frac{d \xi_{y m}}{d \omega}=\frac{\mathbf{v}^{L}{ }_{m} \frac{d \mathbf{A}}{d \omega} \mathbf{v}^{R}}{\mathbf{v}^{L}{ }_{m} \mathbf{B} \mathbf{v}^{R}{ }_{m}}
\end{aligned}
$$

Differentiating Eq. (47) with $\omega$ gives

$$
\xi_{y m} \frac{d \xi_{y m}}{d \omega}+\xi_{z m} \frac{d \xi_{z m}}{d \omega}=\xi_{f} \frac{d \xi_{f}}{d \omega}
$$

Using the relation of $d \xi_{f} / d \omega=1 / c_{f}=\xi_{f} / \omega$, Eq. (65) becomes

$$
\frac{d \xi_{z m}}{d \omega}=\frac{\frac{\xi_{f}^{2}}{\omega}-\xi_{y m} \frac{d \xi_{y m}}{d \omega}}{\xi_{z m}} .
$$


Group velocities of Lamb waves propagating in the $z$ direction are

$$
c_{g m}=\frac{d \omega}{d \xi_{z m}}=\frac{\xi_{z m}}{\frac{\xi_{f}^{2}}{\omega}-\xi_{y m} \frac{d \xi_{y m}}{d \omega}} .
$$

Namely, the group velocity Eq. (67) can be calculated from Eqs. (60), (61), and (64) using eigenvalues $\xi_{y m}, \xi_{z m}$, right and left eigenvectors $\mathbf{v}^{R}{ }_{m}$ and $\mathbf{v}^{L}{ }_{m}$.

\section{Calculation results}

\subsection{Validation of calculation by comparing with theoretical solutions and by checking energy balance}

Solving the eigenvalue problem Eq. (56) yields eigenvalues $\xi_{y m}(m=1$, $2 \ldots 4 N+2)$ and then the wavenumber $\xi_{z m}$ is obtained by the following equation derived from Eq. (47).

$$
\xi_{z m}= \pm \sqrt{\xi_{f}^{2}-\xi_{y m}^{2}}
$$

A pair of positive and negative values stands for a pair of the $m$ th modes of Lamb wave propagating in the $\pm z$ directions. Solid lines in Fig. 3 (a) and (b) are frequency spectra of real and imaginary parts of wavenumber $\xi_{z m}$ for leaky Lamb modes obtained with Eq. (45). The number of layered elements $M$ was set to 64 in this study. Calculation results with the SAFE indicated (solid lines) agree well with theoretical solutions (circles) obtained in Ref. [5] by Chimenti and Rocklin, which designates that the calculation with the SAFE is carried out with sufficiently high accuracy.

A displacement distribution vector $\boldsymbol{\varphi}_{m}$ in Eq. (48) is obtained from a right eigenvector $\mathbf{v}^{R}{ }_{m}$ that is calculated by solving the eigenvalue problem Eq. (56) for each 
mode. Then, substituting a displacement distribution vector $\boldsymbol{\varphi}_{m}$ to the nodal displacement vector in Eq. (3) or (30) gives a displacement vector $\mathbf{u}$ at an arbitrary position and time for the $m$ th mode. Fig. 4 shows a displacement distribution for a mode at (A) in dispersion curve of Fig. 3 in the area of $4 d \times 5 d$ at a certain time, where displacements are magnified to show the wave motion clearly. Color in the figure represents the displacement in the $y$ direction, which proves that displacement in the $y$ direction is continuous at the boundary of the plate and water.

The point (A) in Fig. 3 (a) corresponds to two conjugate solutions $\xi_{z m}$ and $\xi_{z m}{ }^{*}$, which are calculated from a solution pair of $\xi_{y m}$ and $-\xi_{y m}{ }^{*}$ in Eq. (56). Namely, the possible solution at the point (A) are

$$
\begin{array}{ll}
\xi_{y m}=\xi_{y m}{ }^{R}-i \xi_{y m}{ }^{I}, & \xi_{z m}=\xi_{z m}{ }^{R}+i \xi_{z m}{ }^{I} \\
-\xi_{y m}{ }^{*}=-\xi_{y m}{ }^{R}-i \xi_{y m}{ }^{I}, & \xi_{z m}{ }^{*}=\xi_{z m}{ }^{R}-i \xi_{z m}{ }^{I} .
\end{array}
$$

where $\xi_{y m}{ }^{R} \geq 0, \xi_{y m}{ }^{I} \geq 0, \xi_{z m}{ }^{R} \geq 0, \xi_{z m}{ }^{I} \geq 0$. Eq. (69) stands for a mode leaking out from the plate to the water and attenuating as it propagates due to the leakage (Fig. 4 (a)), while Eq. (70) is a mode propagating inward from the water to the plate and amplifying as it propagates due to the incident waves (Fig. 4 (b)).

From displacements at an arbitrary position and time, we can calculate velocity, strain, stress as well as energy flux using the SAFE. Now we calculate energy flux across the plate cross-section $\Gamma_{0}$ and $\Gamma_{1}$ and the plate-water boundaries with the length of the plate thickness $d, \Gamma_{2}$ and $\Gamma_{3}$, as shown in Fig. 5. Fig. 6 shows frequency dependence of energy flux rate $P_{1}\left(=E_{1} / E_{0}\right), P_{2}\left(=E_{2} / E_{0}\right)$, and $P_{3}\left(=E_{3} / E_{0}\right)$, where $E_{0}, E_{1}, E_{2}$, and $E_{3}$ are energy flux across the boundaries $\Gamma_{0}, \Gamma_{1}, \Gamma_{2}$, and $\Gamma_{3}$, respectively, taking their positive in the arrow direction in Fig. 5. 
Since Fig. 6 (a) shows a mode that flows into from $\Gamma_{0}$ and leaks out from $\Gamma_{1}, \Gamma_{2}$, and $\Gamma_{3}$, the energy flux rate $P_{1}, P_{2}, P_{3}$ are positive and the energy flux leaking out from the upper and lower boundaries $\Gamma_{1}$ and $\Gamma_{2}$ are identical. Moreover, $P_{1}+P_{2}+P_{3}=1$ showing the natural consequences that the energy flowing into at $\Gamma_{0}$ leaks out from $\Gamma_{1}, \Gamma_{2}$ and $\Gamma_{3}$. While, in the increasing mode (Fig. 6 (b)), the energy fluxes at $\Gamma_{2}$ and $\Gamma_{3}\left(P_{2}, P_{3}\right)$ are negative, which shows that energy flows into at the upper and lower boundaries. And the energy flux across $\Gamma_{1}$ is larger than the input energy at $\Gamma_{0}$.

\subsection{Characteristic properties on leaky guided waves}

Fig. 7 shows frequency spectra of wavenumber (real and imaginary parts), phase velocity, and group velocity calculated with the SAFE for four different cases. Black lines in Figs. 7 (a) - (d) denote the case without leaky media $\left(\rho_{1}=\rho_{2}=0\right.$ in Eqs. (44) or (45)), and blue lines are total transmission modes in the same leaky media as $\rho_{1}=\rho_{2}$ in Eq. (44), and red lines are leaky plate modes in the same leaky media as $\rho_{1}=\rho_{2}$ in Eq. (45), and gray lines represents the case when leaky liquid medium exists at one side by setting $\rho_{1}=0$. Unnecessary equations and unknown valuables appearing in Eq. (56) for $\rho_{1}=0$ and/or $\rho_{2}=0$ were removed before solving the eigenvalue problem.

When water exists as leaky media, some characteristic solutions were observed. The first one is that a mode with phase and group velocities slightly smaller than the velocity of water, $1500 \mathrm{~m} / \mathrm{s}$, appears only when water exists. The second feature is that group velocity dispersion curves are significantly distorted in the frequency range where the phase velocities of $\mathrm{A} 0$ and $\mathrm{S} 0$ modes become close in total transmission modes. The third one is that attenuation curves are significantly different in the conditions of leaky media. These three features are discussed below in detail. 
3.2.1 Non-dispersive modes with a velocity close to that of a leaky medium

Fig. 8 shows a displacement distribution at (B) in Fig. 7 (c), where the phase velocity is $c_{p}=\omega / \operatorname{Re}\left(\xi_{z}\right)=1496.4 \mathrm{~m} / \mathrm{s}$, and wavenumbers in the $y$ and $z$ directions are a pure imaginary number $1.16 \times 10^{3} i$ and a real number $1.68 \times 10^{4}$, respectively. As this mode propagates in the $z$ direction with the energy distribution localized on the solid fluid boundaries, its attenuation, the imaginary part of $\xi_{z}$, becomes zero. This mode is known as Scholte waves propagating on solid-liquid boundaries. Since this mode also has an energy distribution within a solid at the vicinity of the boundaries, the localized energy distribution affects the opposite boundary gradually as the plate becomes thinner. Therefore, the Scholte mode exhibit a non-flat frequency range $f d=0.5 \mathrm{MHz} \mathrm{mm}$ in Fig. $7(\mathrm{c})$.

\subsubsection{A0 and S0 total transmission modes}

In total transmission modes designated in blue lines in Fig. 7, distinctive features were exhibited at the range of $f d=3-5 \mathrm{MHz} \mathrm{mm}$ where phase velocities of A0 and S0 modes become close. Phase and group velocity dispersion curves, Figs. 7 (a) and (b) are zoomed around $f d=3-5 \mathrm{MHz} \mathrm{mm}$ as shown in Figs. 9 (a) and (b). The A0 and S0 modes below about $f d=3.78 \mathrm{MHz}$ mm show similar curves with the cases of non-leaky media and leaky modes, while the curves of A0 and S0 modes merge together at $f d=$ 3.78 $\mathrm{MHz} \mathrm{mm}$ and vary with the identical phase and group velocities beyond the $f d$ value.

Fig. 10 represents displacement distributions of four modes calculated for the point $(C)$ in Fig. $9(f d=5)$. Figs. 10 (a) and (b) are modes with large vibration at a single side of the plate-water boundaries from which leaky wave flows out, while in (c) 
and (d), incident wave flows into from a large vibrating boundary. Both (a), (b) and (c), (d) are symmetric with respect to the centerline of the plate, from which we can infer their phase and group velocities are identical. These modes correspond to the solution

pair of $\xi_{y m}$ and $-\xi_{y m}{ }^{*}$ as given in Eqs. (69) and (70). Although these fundamental Lamb wave modes are far from antisymmetric and symmetric distributions, we call them $\mathrm{A} 0$ and $\mathrm{S} 0$ for the sake of convenience.

Around the frequency range where curves of S0 and A0 modes merge, group velocity dispersion curves become discontinuous. We consider that this feature is caused by non-smooth variation of attenuation coefficient as shown in Fig. 7 (b) and Fig. 11. Because the attenuation curves suddenly vary from zero to a certain value at the junction of S0 and A0 modes, the differentiation of the curve, corresponding to group velocity, becomes discontinuous.

\subsubsection{Attenuation curves}

Fig. 7 (b) shows that attenuation coefficients significantly differ in boundary conditions. For the case of non-leaky media (black lines), attenuation coefficients have non-zero values only below the cutoff frequencies of $\mathrm{A} 1$ and $\mathrm{S} 1$ modes, showing evanescent modes. Modes with leaky media also have almost identical values with the attenuation coefficients of evanescent modes, and therefore blue, red, and gray lines are behind the black line in Fig. 7 (b).

Fig. 11 shows attenuation curves only for A0 (solid line) and S0 (dashed line) modes in a water-coupled plate. As shown in the previous section, attenuation curves abruptly change from zero to a non-zero value at $f d=3.78 \mathrm{MHz} \mathrm{mm}$ for total transmission modes and the attenuation coefficients for A0 and S0 modes are identical, which corresponds to Figs. 10 (a) and (b).

For leaky plate modes, attenuation in a water-loaded plate on both two sides 
(red lines) is approximately double that in a one sided plate (gray lines) below about $f d$ $=3 \mathrm{MHz} \mathrm{mm}$. This is caused by the Lamb wave feature that A0 and S0 modes in the low frequency range propagate with almost uniform displacement distributions in the thickness direction with or without leaky media as shown in Fig. 12. As ultrasonic energy leak out from a single side in Fig. 12 (b), attenuation became half of that in a water - loaded plate on both boundaries (Fig. 12(a)). While, in the high frequency range as shown in Fig. 11 (E), displacement distributions were obtained as Fig. 13. When leaky media exist at both sides of boundaries, the S0 mode kept symmetric distribution with respect to the plate center (Fig. 13 (a)), while for a water-loaded plate on a single side, the "S0 mode" have significantly different displacement distribution where vibration is localized at the free boundary (Fig. 13(b)). This unique feature results in reducing attenuation as shown in the solid gray line of Fig. 11 over $f d=4 \mathrm{MHz} \mathrm{mm}$.

\section{Conclusions}

This paper described calculation of Lamb waves in a plate surrounded by leaky media using a SAFE. The SAFE formulation provided a nonlinear eigenvalue problem for leaky Lamb waves in a plate coupled with leaky media including elastic solids and fluids. The nonlinear eigenvalue problem, obtained for a plate coupled with leaky media, was deformed into the linear eigenvalue problem using the symmetry of Lamb wave modes under the conditions that leaky media are non-viscous fluids with the same sound velocities. Dispersion curves calculated with the SAFE agreed well with theoretical solutions, which proves the validity and accuracy of the SAFE calculations. Moreover, we discussed displacement distributions for characteristic modes strongly affected by leaky media. 


\section{Acknowledgments}

The authors would like to thank Professor Shiro Biwa for his insightful comments and continuing support.

\section{References}

[1] I. A. Viktrov, Rayleigh and Lamb waves, Plenum press, New York, 1967

[2] J. L. Rose, Ultrasonic waves in solid media, Cambridge University Press, 1999

[3] M. F. M. Osborne, S.D. Hart, Transmission, reflection, and guiding of an exponential pulse by a steel plate in water. I. Theory, Journal of the Acoustical Society of America. 17 (1945) 1-18.

[4] S. I. Rokhlin, D.E. Chimenti, A.H. Nayfeh, On the topology of the complex wave spectrum in a fluid-coupled, Journal of the Acoustical Society of America. 85 (1989) $1074-1080$.

[5] D. E. Chimenti, S.I. Rokhlin, Relationship between leaky Lamb modes and reflection coefficient zeroes for a fluid-coupled elastic layer, Journal of the Acoustical Society of America. 88 (1990) 1603-1611.

[6] V. Dayal, K. Vikram K, Leaky Lamb waves in an anisotropic plate. I: An exact solution and experiments, Journal of the Acoustical Society of America. 85 (1989) $2268-2276$.

[7] M. J. S. Lowe, Matrix techniques for modeling ultrasonic waves in multilayered media, IEEE Transactions on Ultrasonics, Ferroelectrics and Frequency Control. 42 (1995) 525-542.

[8] B. Pavlakovic, M.J.S. Lowe, D.N. Alleyne, P. Cawley, Disperse: a general purpose program for creating dispersion curves, in: D.O. Thompson, D.E. Chimenti (Eds.), Review of Progress in Quantitative NDE, vol. 16, Plenum Press, New York, 1997, pp. 
$185-192$.

[9] G. R. Liu, J.D. Achenbach, Strip element method for stress analysis of anisotropic linearly elastic solids, Journal of Applied Mechanics. 61 (1994) 270 - 277.

[10] L. Gavrić, Computation of propagative waves in free rail using a finite element technique, Journal of s Sound Vibration. 185 (1995) 531-543.

[11] L. Gry, Dynamic modelling of railway track based on wave propagation, J. Sound Vibration. 195 (3) (1996) 477-505.

[12] D. J. Thompson, C.J.C. Jones, Sound radiation from a vibrating railway wheel, J. Sound Vibration. 253 (2) (2002) 401-419.

[13] T. Hayashi, K. Kawashima, Multiple reflections of Lamb waves at a delamination, Ultrasonics. 40 (2002) 193-197.

[14] T. Hayashi, W - J Song, J. L. Rose, Guided wave dispersion curves for a bar with an arbitrary cross-section, a rod and rail example. Ultrasonics. 41 (2003) 175-183.

[15] T. Hayashi, J. L. Rose, Guided wave simulation and visualization by a semi-analytical finite element method, Materials Evaluation. 61 (1) (2003) 75-79.

[16] T. Hayashi, C. Tamayama, M. Murase, Wave structure analysis of guided wave in a bar with an arbitrary cross-section, Ultrasonics. 44 (1) (2006) 17-24.

[17] T. Hayashi, K. Kawashima, J. L. Rose, Calculation for guided waves in pipes and rails, Key Engineering Materials. 270-273 (2004) 410-415.

[18] T. Hayashi, K. Kawashima, Z. Sun, J. L. Rose, Guided wave propagation mechanics across a pipe elbow, Journal of Pressure Vessel Technology, 127 (3) (2005) $322-327$.

[19] I. Bartoli, A. Marzani, F. Lanza di Scalea, E. Viola, Modeling wave propagation in damped waveguides of arbitrary cross-section, Journal of Sound and Vibration. 295 (2006) 685-707. 
[20] P.W. Loveday, Semi-analytical finite element analysis of elastic waveguides subjected to axial loads., Ultrasonics. 49 (2009) 298-300.

[21] M. Castaings, M. Lowe, Finite element model for waves guided along solid systems of arbitrary section coupled to infinite solid media., The Journal of the Acoustical Society of America. 123 (2008) 696-708.

[22] S. Banerjee, T. Kundu, Semi-analytical modeling of ultrasonic fields in solids with internal anomalies immersed in a fluid, Wave Motion. 45 (2008) 581-595.

[23] M. Mazzotti, I. Bartoli, a. Marzani, E. Viola, A coupled SAFE-2.5D BEM approach for the dispersion analysis of damped leaky guided waves in embedded waveguides of arbitrary cross-section, Ultrasonics. 53 (7) (2013) 1227 - 1241. 


\section{Figure 1}

Layered elements and leaky media in the SAFE calculation.

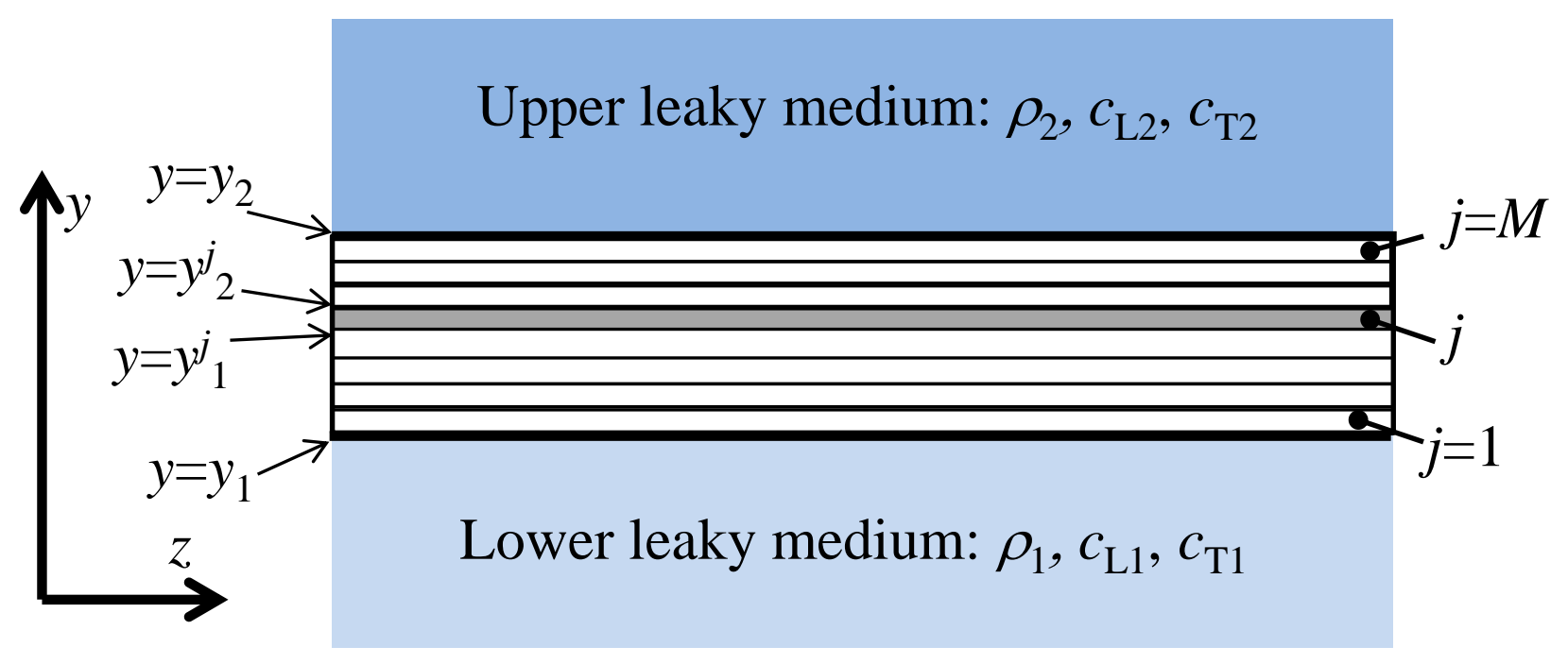




\section{Figure 2}

Wavenumbers and vibration directions.
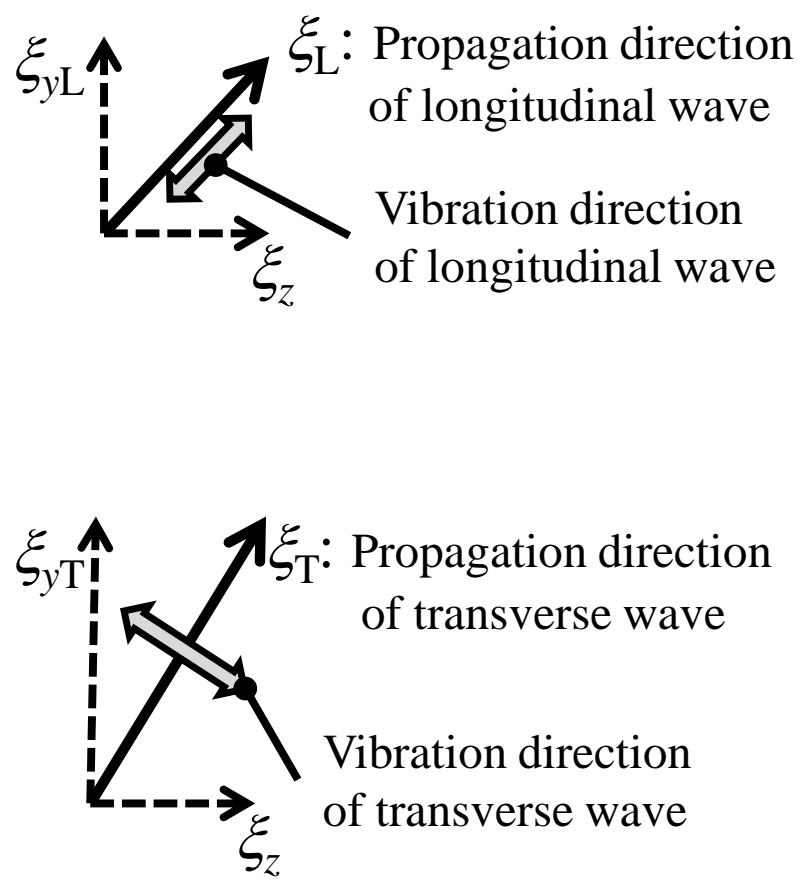


\section{Figure 3}

Comparison of dispersion curves for leaky plate modes with the previous theoretical solutions

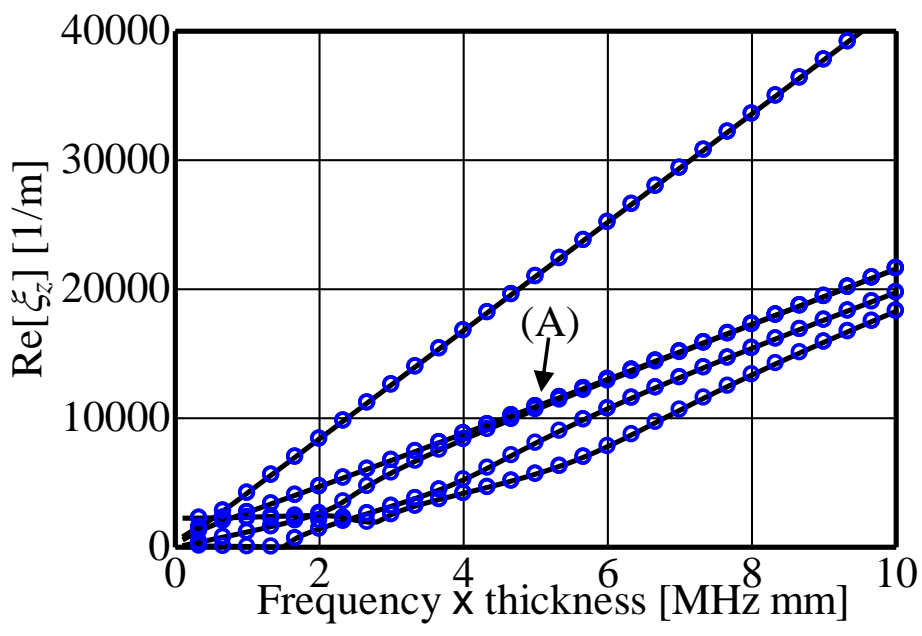

(a) Wave number $\operatorname{Re}\left(\xi_{z m}\right)$

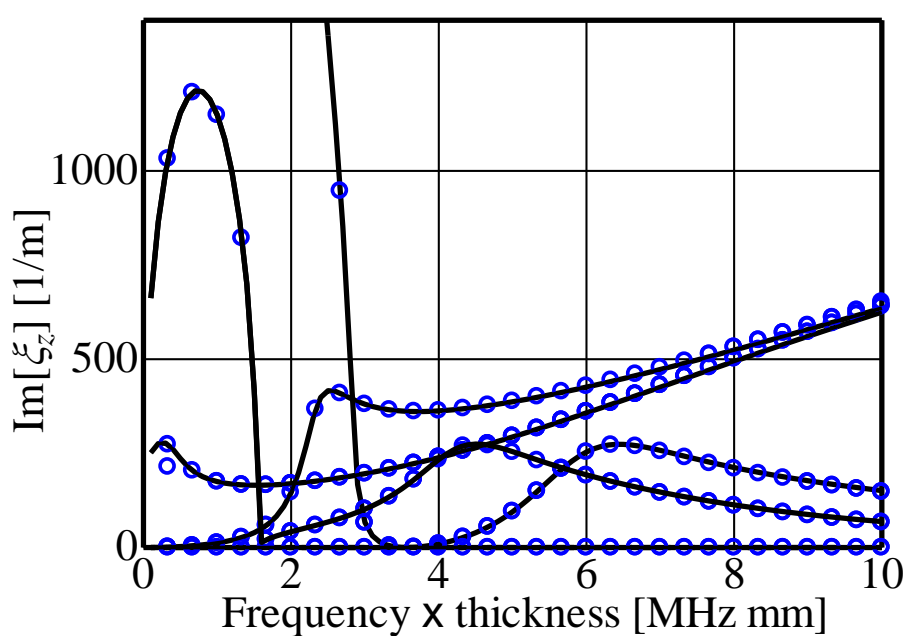

(b) Attenuation $\left(\operatorname{Im}\left(\xi_{z m}\right)\right)$ 


\section{Figure 4}

Displacement distributions at the point (A) in Fig.3(a).
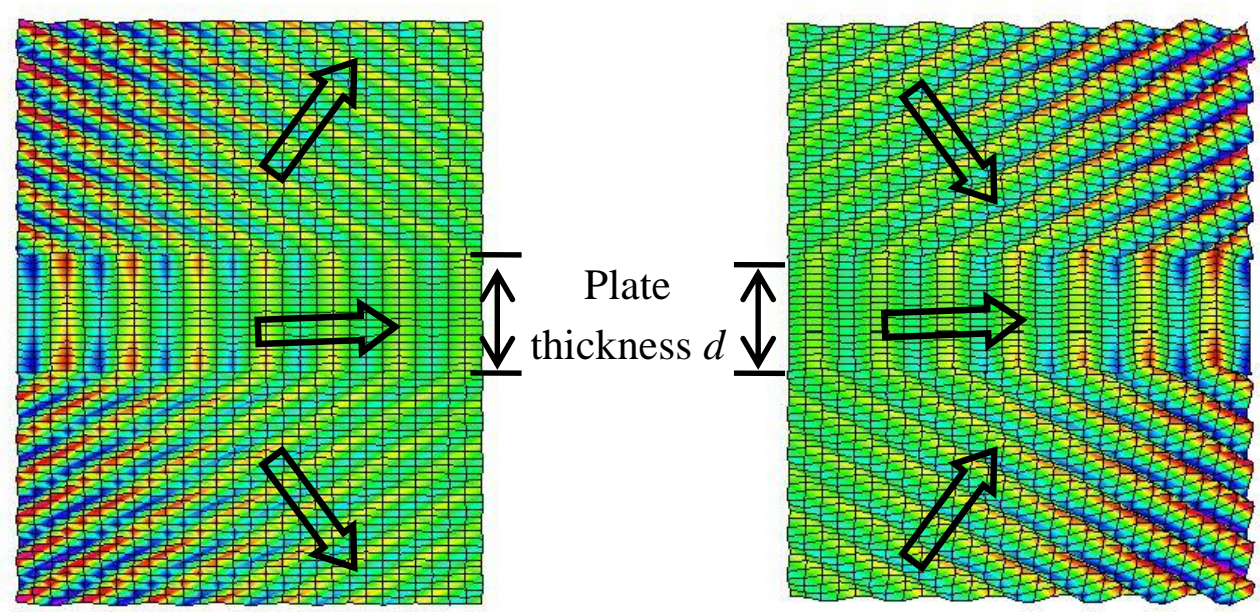

(a) Attenuated mode

with outgoing leaky wave

(b) Increasing mode

with incoming oblique incidence

\section{Figure 5}

Energy flow at the boundaries.

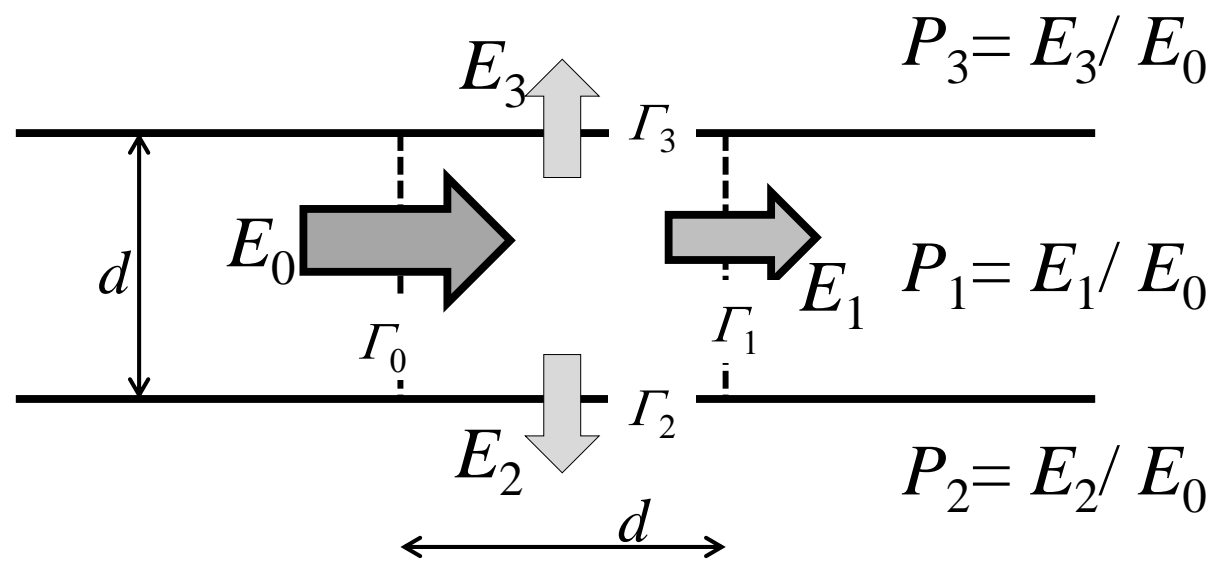




\section{Figure 6}

Energy flow rate for the mode including the point (A).

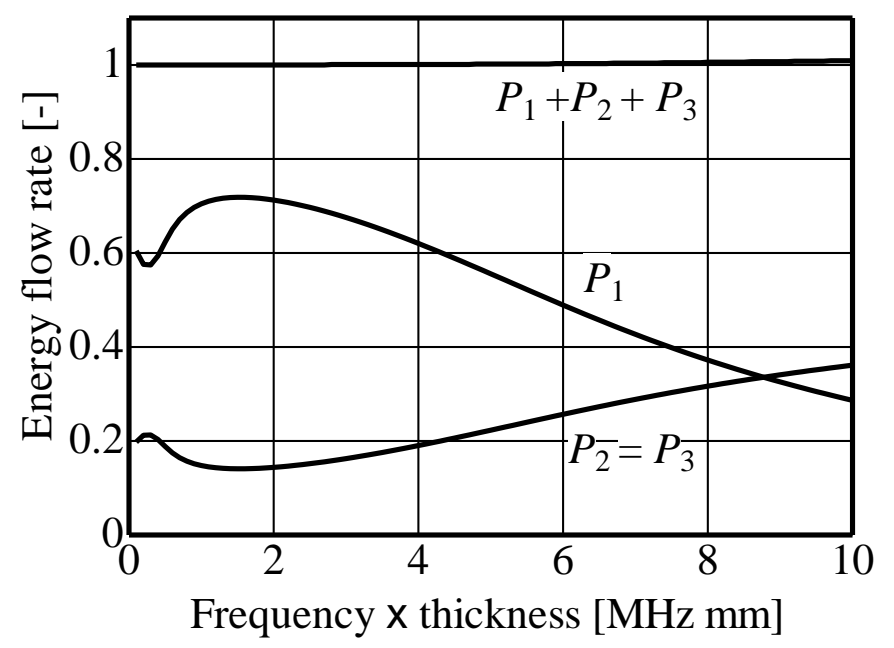

(a) Attenuated mode with outgoing leaky wave

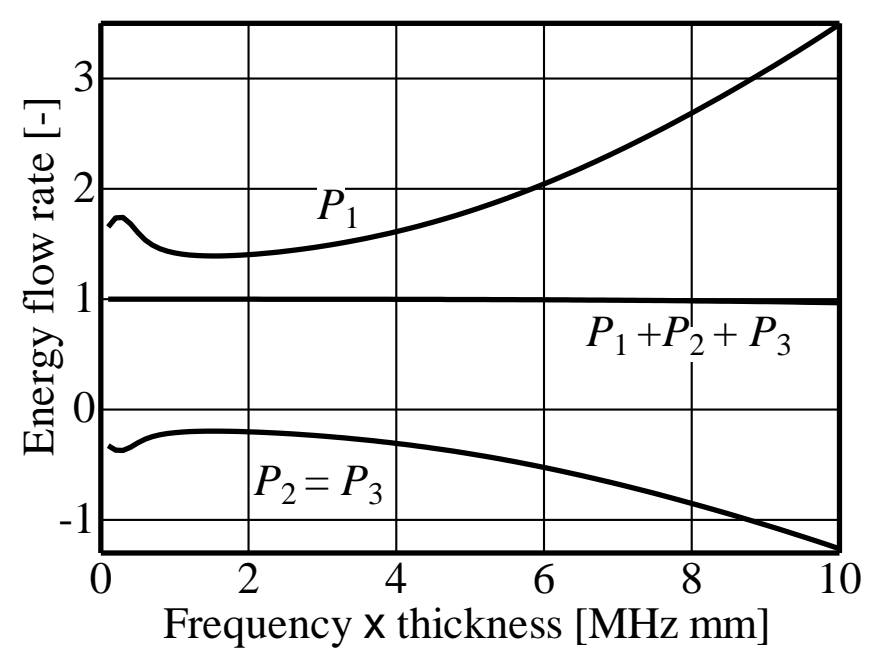

(b) Increasing mode

with incoming oblique incidence 


\section{Figure 7}

Frequency spectra for water-loaded and unloaded aluminum plates.

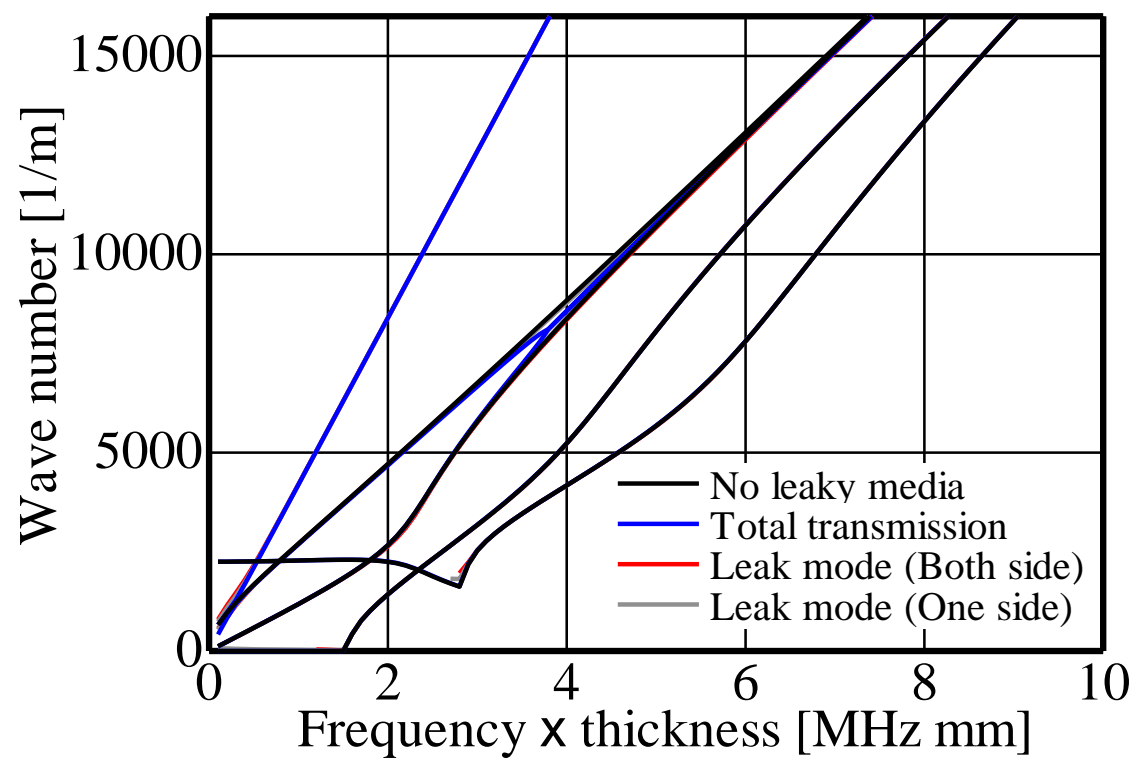

(a) Wave number $\left(\operatorname{Re}\left(\xi_{z m}\right)\right)$

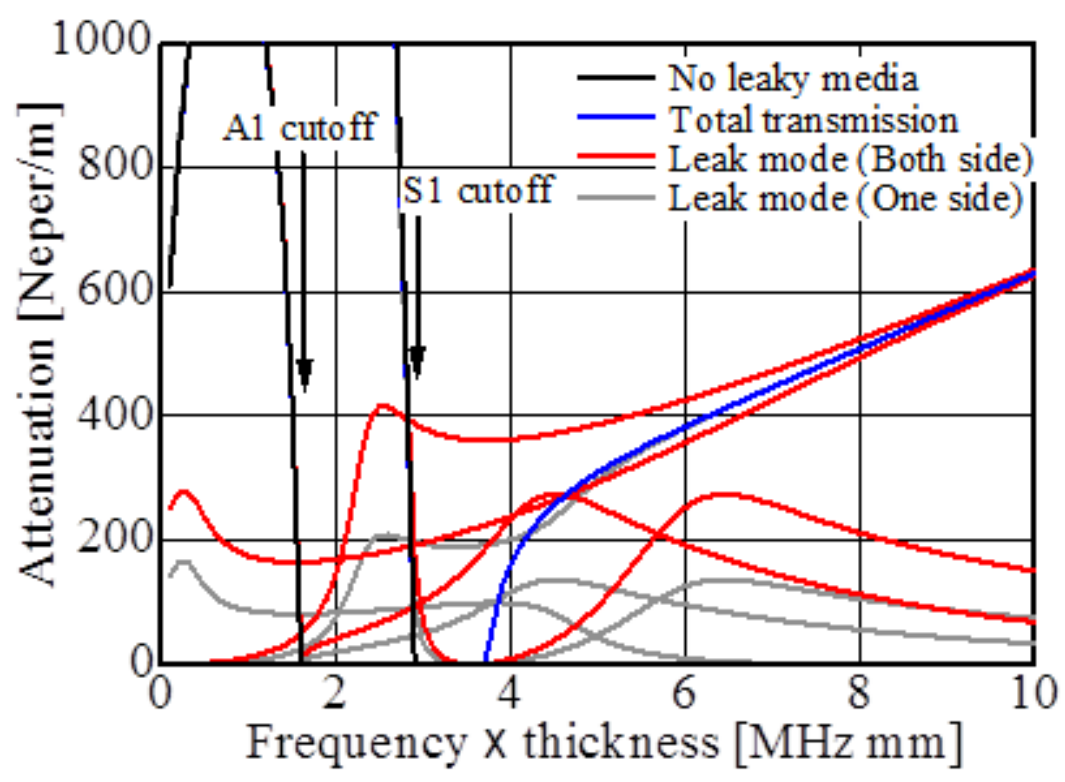

(b) Attenuation coefficient $\left(\operatorname{Im}\left(\xi_{z m}\right)\right)$ 
Figure 7

(contd.)

Frequency spectra for water-loaded and unloaded aluminum plates.

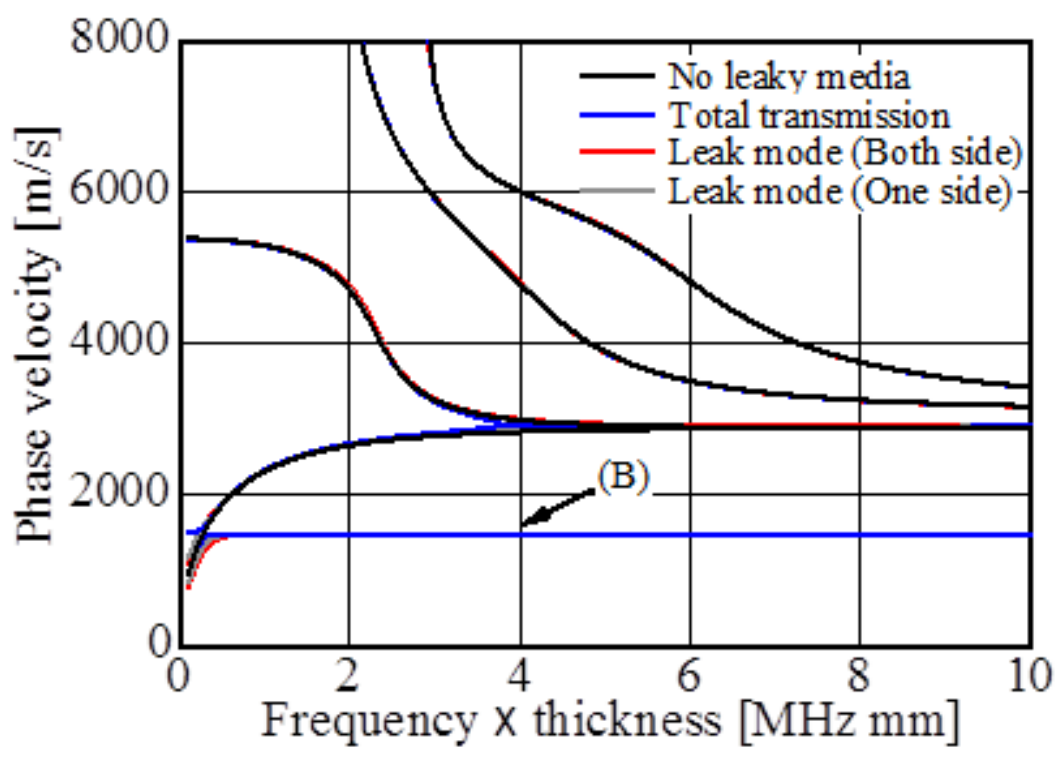

(c) Phase velocity dispersion curves $\left(c_{p}=\omega / \operatorname{Re}\left(\xi_{z}\right)\right)$

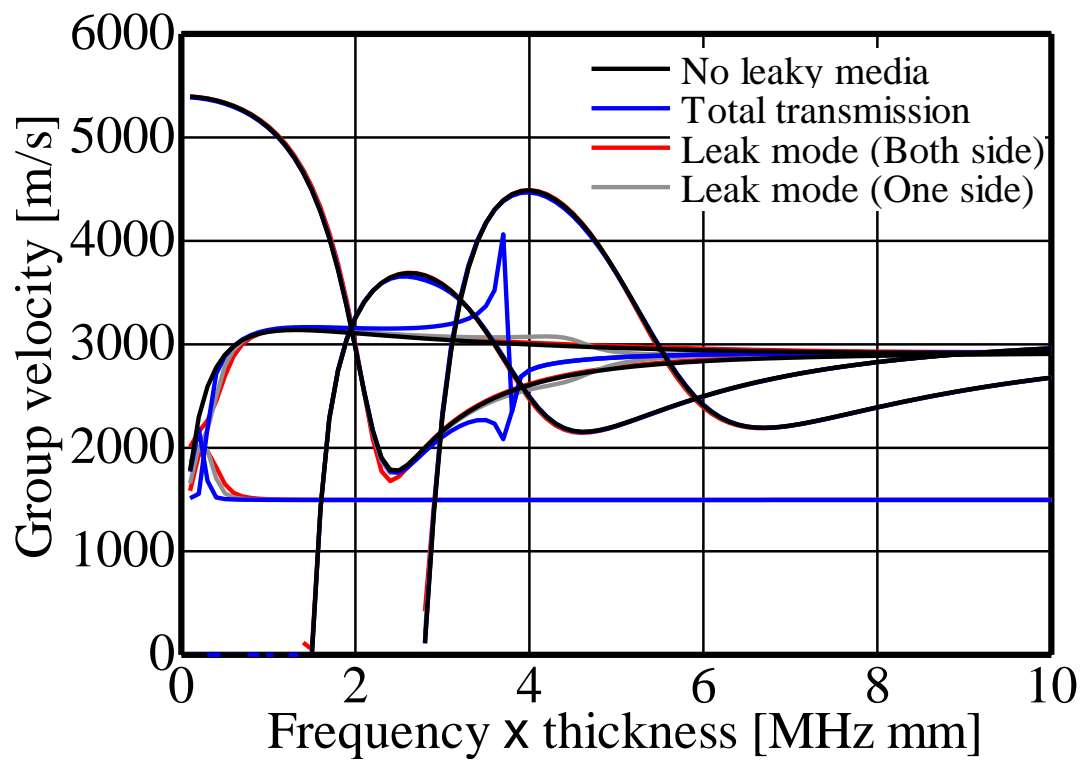

(d) Group velocity dispersion curves 


\section{Figure 8}

Wave structure at the point (B) in Fig.7 (c).

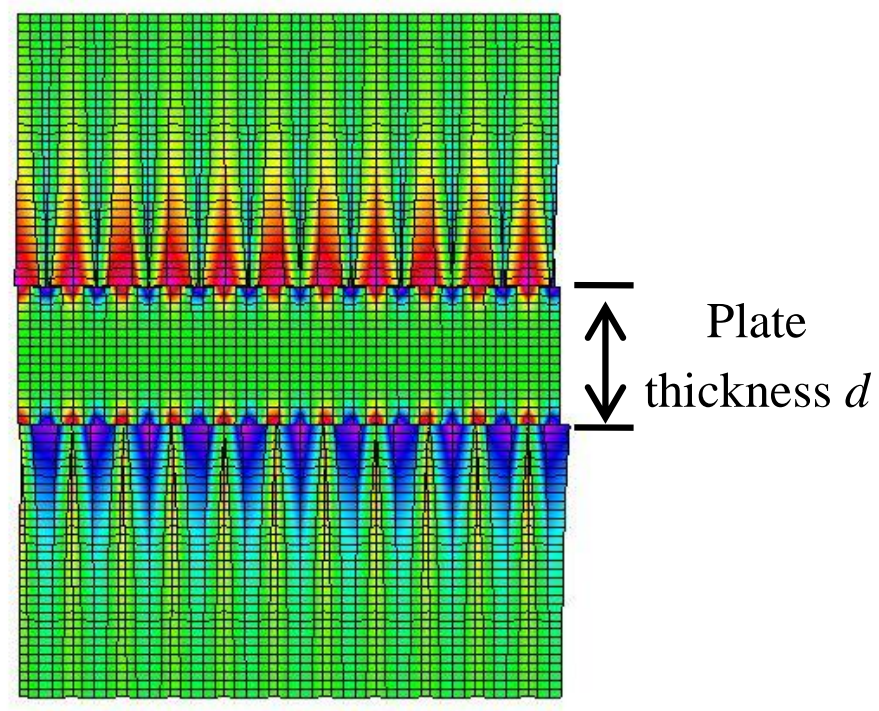


Figure 9

A0 and S0 mode dispersion curves for four different boundary conditions.

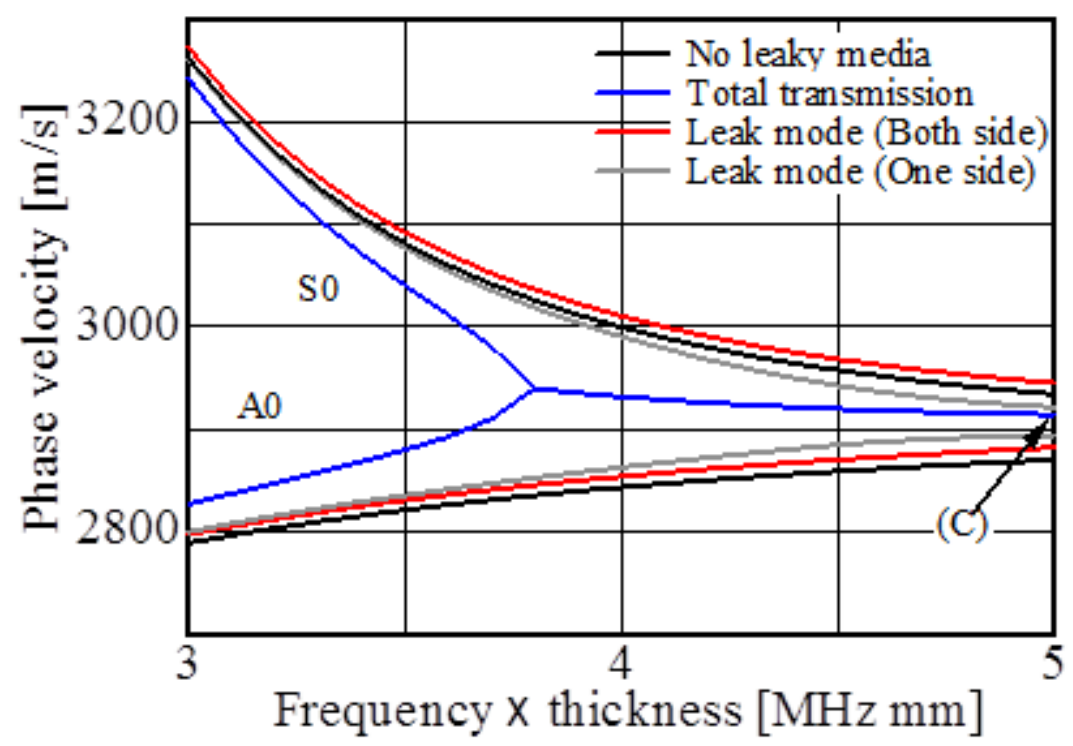

(a) Phase velocity

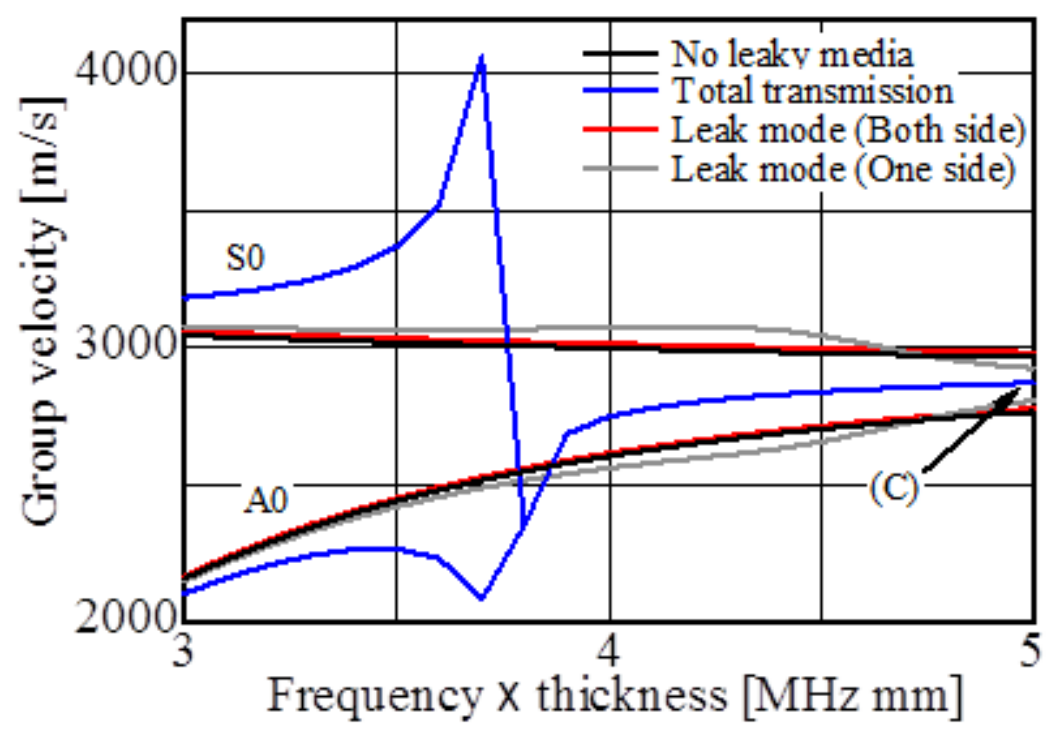

(b) Group velocity 


\section{Figure 10}

Displacement distributions of four modes calculated for the point (C) in Fig. $9(f d=5)$

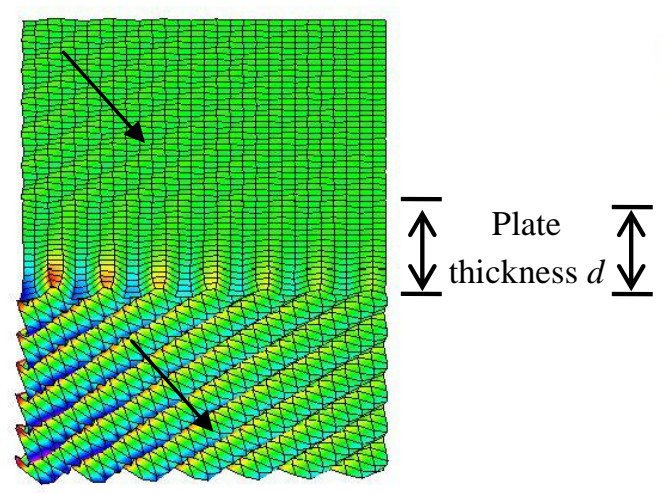

(a) Solution 1

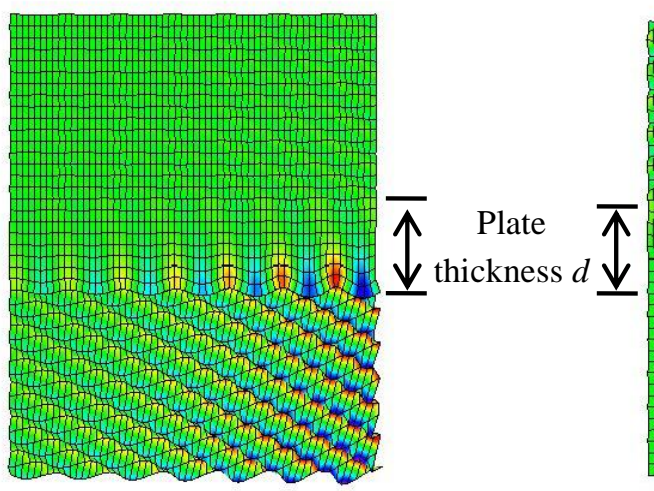

(c) Solution 3

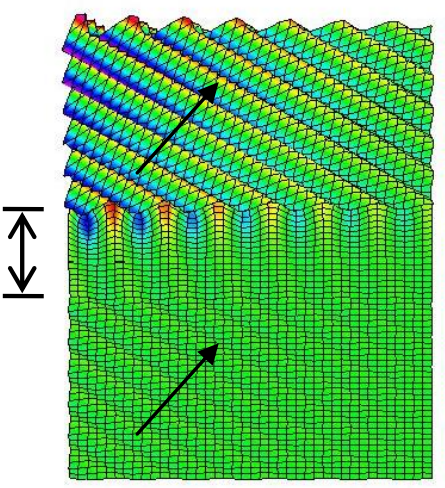

(b) Solution2

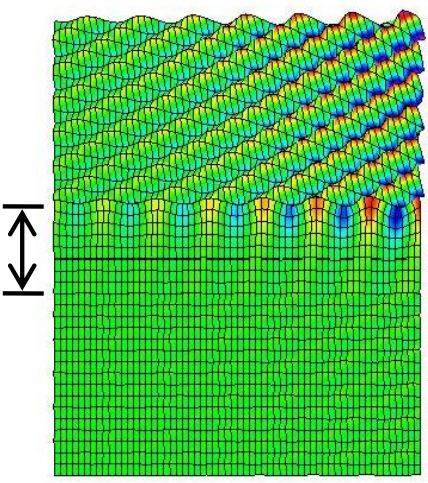

(d) Solution4 
Figure 11

Attenuation curves for A0 and S0 modes in a water-loaded plate.

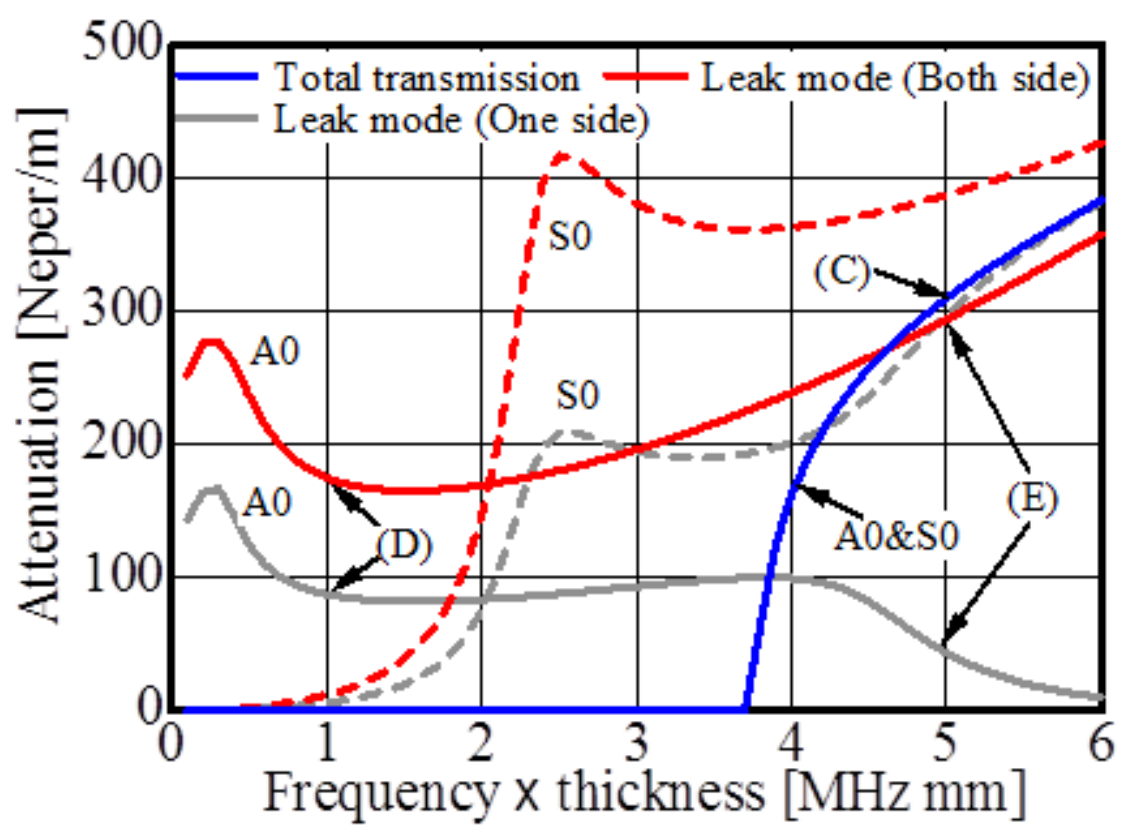




\section{Figure 12}

Displacement distributions for the point (D) in Fig. 11.

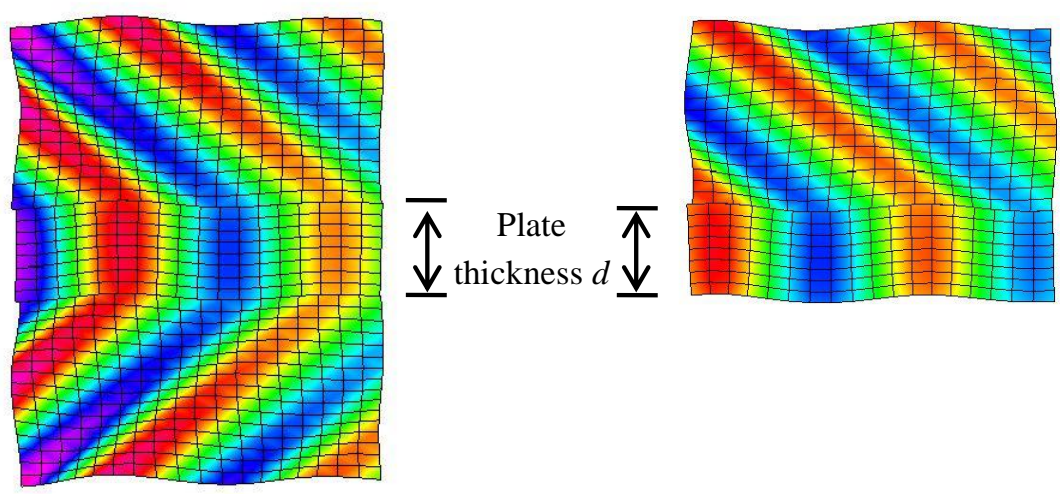

\section{Figure 13}

Displacement distributions for the point (E) in Fig. 11.

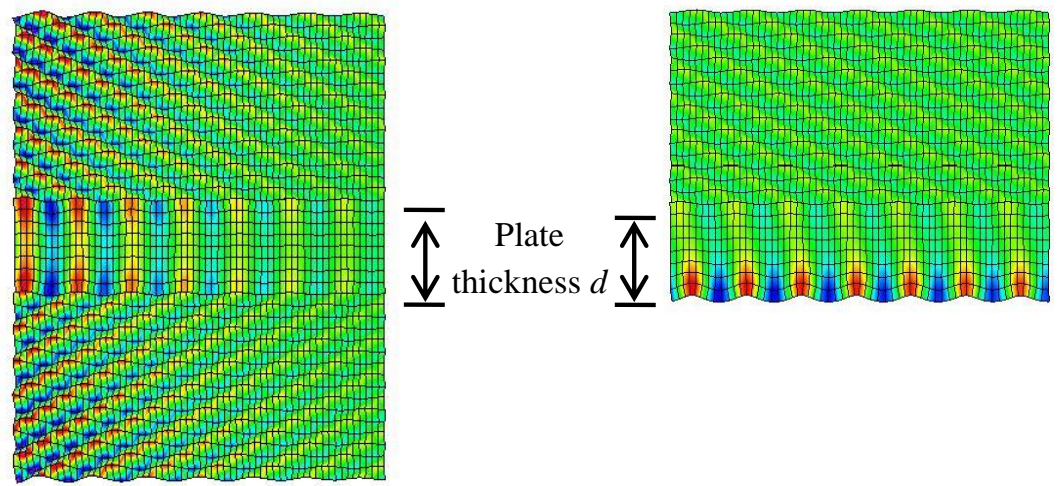


We developed a semi-analytical finite element method for calculating leaky Lamb waves. The calculation results agreed well with the previous theoretical studies. We discussed dispersion curves, attenuation curves, and displacement distributions for total transmission modes and leaky plate modes in a single side and both two side water-loaded plate 